\title{
Mitotic replisome disassembly in vertebrates
}

Sara Priego Moreno ${ }^{1,2}$, Rebecca M. Jones ${ }^{1}$, Divyasree Poovathumkadavil ${ }^{1}$, Agnieszka Gambus $^{1}$

1. Institute for Cancer and Genomic Sciences, College of medical and Dental Sciences, University of Birmingham, Edgbaston, Birmingham, B15 2TT, UK

2. Present address: Salk Institute for Biological Studies, 10010 N Torrey Pines Rd, La Jolla, California 92037

\section{ABSTRACT}

Recent years have brought a breakthrough in our understanding of the process of eukaryotic DNA replication termination. We have shown that the process of replication machinery (replisome) disassembly at the termination of DNA replication forks in S-phase of the cell cycle is driven through polyubiquitylation of one of the replicative helicase subunits Mcm7. Our previous work in C.elegans embryos suggested also an existence of a back-up pathway of replisome disassembly in mitosis. Here we show, that in Xenopus laevis egg extract, any replisome retained on chromatin after S-phase is indeed removed from chromatin in mitosis. This mitotic disassembly pathway depends on formation of K6 and K63 ubiquitin chains on Mcm7 by TRAIP ubiquitin ligase and activity of p97/VCP protein segregase. The mitotic replisome pathway is therefore conserved through evolution in higher eukaryotes. However, unlike in lower eukaryotes it does not require SUMO modifications. This process can also remove any helicases from chromatin, including "active" stalled ones, indicating a much wider application of this pathway than just a "back-up" for terminated helicases.

\section{INTRODUCTION}

Faithful cell division is the basis for the propagation of life and requires accurate duplication of all genetic information. DNA replication must be precisely regulated as unrepaired mistakes can change cell behaviour with potentially severe consequences, such as genetic disease, cancer and premature ageing (Burrell, McClelland et al., 2013). Fundamental studies have led to a step-change in our understanding of the initiation of DNA replication and DNA synthesis, but until discovery of the first elements of the eukaryotic 
1 replisome disassembly mechanism in 2014 , the termination stage of eukaryotic replication was mostly unexplored.

DNA replication initiates from thousands of replication origins. They are the positions within the genome where replicative helicases become activated and start unwinding DNA while moving in opposite directions, away from each other, creating two DNA replication forks. The replicative helicase is composed of Cdc45, Mcm2-7 hexamer and GINS complex (CMG complex) (Moyer, Lewis et al., 2006); it is positioned at the tip of replication forks and forms a platform for replisome assembly (Replisome Progression Complex, RPC) (Gambus, Jones et al., 2006). Once established, the replication forks replicate chromatin until they encounter forks coming in opposite directions from neighboring origins. At this point the termination of replication forks takes place. As CMG helicases travel on the leading strand templates at the forks, the strand encircled by converging helicases differs due to the antiparallel nature of the DNA molecule (Fu, Yardimci et al., 2011). The two converging helicases can therefore pass each other allowing for completion of DNA synthesis. Finally, removal of the replisome from fully duplicated DNA is the last stage of forks termination (Dewar, Budzowska et al., 2015). We have shown that in Xenopus laevis egg extract and in C. elegans embryos this replisome removal in S-phase is driven by Cul2 ${ }^{\mathrm{LRR} 1}$ ubiquitin ligase, which ubiquitylates Mcm7 within the terminated CMG complex (Sonneville, Moreno et al., 2017). Such modified CMG is then recognized by p97 (VCP) segregase and removed from chromatin allowing for disassembly of the whole replisome built around the helicase (Moreno, Bailey et al., 2014).

Interestingly, we have shown that in $C$. elegans embryos, any helicase complexes that fail to be unloaded in S-phase are alternatively unloaded in prophase of mitosis (Sonneville et al., 2017). This potential back-up mechanism functions when CUL-2 ${ }^{\mathrm{LRR}-1}$ activity is blocked and, like the S-phase pathway, depends on the p97 segregase for unloading. Unlike the Sphase pathway, however, it requires an additional p97 cofactor UBXN-3/FAF1 and the SUMO-protease ULP-4 (Senp6/7 homologue in higher eukaryotes) (Sonneville et al., 2017). Interestingly, budding yeast do not possess this mitotic replisome disassembly pathway; cells lacking SCF ${ }^{\mathrm{Dia} 2}$ activity, the ubiquitin ligase responsible for $\mathrm{Mcm} 7$ ubiquitylation in $S$. cerevisiae, accumulate post-termination replisomes on DNA until the next G1 of the next cell cycle (Maric, Maculins et al., 2014). Our aim therefore was to determine if this mitotic replisome disassembly pathway has been conserved throughout evolution and is functioning in higher eukaryotes, or if it is a phenomenon specific to $C$. elegans embryos. Here we show that a mitotic disassembly pathway does exist in vertebrates and determine the first elements of its regulation. We show that only a restricted part of the replisome stays retained on chromatin throughout mitosis in Xenopus egg extract. The disassembly of this replisome is independent of cullin type ubiquitin ligases but requires p97 segregase function. Mitotic 
1 replisome disassembly depends on K6 and K63 ubiquitin chains but not SUMO modifications. We also identify TRAIP ubiquitin ligase as essential for Mcm7 ubiquitylation in mitosis. Finally, we show that active forms of helicase can also be unloaded in this way, suggesting that rather than being a back-up pathway for the disassembly of terminated replisomes, this process is essential to remove any replisome from chromatin before cell division.

\section{RESULTS}

Xenopus egg extract is a cell-free system, which has proven to be instrumental over the years in studies of DNA replication. To determine the existence of a mitotic replisome disassembly pathway in Xenopus egg extract we needed to allow our extract to progress from interphase into mitosis. Routinely we block this cell cycle transition by inhibition of cyclin synthesis in the extract through supplementation with cycloheximide, since it allows for better synchronization of the replication reaction (Gillespie, Gambus et al., 2012). To allow the extract to progress into mitosis we need therefore to supplement it with cyclins either by addition of mitotic extract or addition of recombinant cyclin. We purified His-tagged Xenopus laevis Cyclin A1 N $\Delta 56$ (hereafter: Cyclin A1 $\Delta$ ) and added it to the extract upon completion of DNA replication, as described previously, to induce mitotic entry (EV Fig 1A) (Strausfeld, Howell et al., 1996). Upon addition of Cyclin A1 $\Delta$ we could see condensation of chromatin into chromosomes - a clear sign of mitosis (EV Fig 1B).

To test if the replisome retained on chromatin in S-phase can be unloaded as cells enter mitosis we followed the experimental path detailed in Fig 1A. A replication reaction was set up in the interphase extract supplemented with Cullin ligase inhibitor MLN4924 to block Cul2 ${ }^{\mathrm{LRR} 1}$ activity and the S-phase replisome unloading (Sonneville et al., 2017). After completion of replication (90 min, EV Fig 1A) we optionally added Cyclin $A 1 \Delta$, isolated chromatin at different time points during mitosis progression and analysed chromatin bound proteins by western blotting (Fig 1B). In the late S-phase samples (buffer control), the CMG helicase (represented by Cdc45 subunit) remained associated with chromatin and Mcm7 displayed low levels of ubiquitylation, as seen before upon MLN4924 treatment and Cul2 immunodepletion (Moreno et al., 2014, Sonneville et al., 2017), however upon addition of Cyclin A1 $\Delta$, Cdc45 and ubiquitylated Mcm7 were efficiently unloaded. This result indicates that indeed the mitotic replisome disassembly pathway is evolutionarily conserved and exists in vertebrates and that, unlike the S-phase pathway, it does not require activity of Cullin type ubiquitin ligases because the Cullin inhibitor was present throughout the reaction (Fig 1B).

Next, we tested whether the mitotic replisome disassembly pathway required the activity of the p97 segregase. We followed the experimental setup as before but now optionally added the inhibitor of $\mathrm{p} 97, \mathrm{NMS} 873$. The retained replisome was unloaded upon Cyclin A1 $\Delta$ 
1 addition in the absence but not in the presence of the p97 inhibitor indicating that indeed p97 does play an essential role in promoting mitotic replisome disassembly (Fig 1C). We could also see an analogous result if the p97 inhibitor was present throughout the two stages of the cell cycle as the only way to block replisome disassembly (EV Fig 1C).

Interestingly, when the mitotic unloading of replisome was blocked with p97 inhibitor, we could clearly see accumulation of highly modified forms of Mcm7 on chromatin (Fig 1C and EV Fig 1C). To examine whether these modifications were due to further ubiquitylation of Mcm7, we blocked S-phase and mitotic replisome disassembly by addition of p97 inhibitor from the beginning of the replication reaction, induced mitosis after completion of DNA sythesis and optionally supplemented extract with a high concentration of wild-type (wt) ubiquitin or a chain-terminating mutant of ubiquitin with all lysines mutated (UbiNOK). Supplementation of mitotic extract with wt ubiquitin allowed for accumulation of highly modified Mcm7 on chromatin in mitosis as before (Fig 1D, left). However, addition of UbiNOK blocked further modifications of $\mathrm{Mcm} 7$, leaving only the chains which were built previously in S-phase (Fig1D, right). To determine whether this further Mcm7 polyubiquitylation in mitosis was essential for mitotic replisome disassembly we repeated the experiment with addition of wt Ubi or UbiNOK but this time only in the presence of the Cullin inhibitor from the start of the reaction (Fig 1E). Addition of UbiNOK to mitotic extract blocked disassembly of the replisome (as shown by permanent Cdc45 chromatin binding) suggesting that further Mcm7 polyubiquitylation is required for its unloading. We could also observe that LRR1 (the substrate specific subunit of Cullin 2, targeting Mcm7 in S-phase) dissociates from chromatin in mitosis irrespectively of replisome disassembly, in agreement with the finding that it does not play an essential role in this pathway (Fig 1D and E) .

As the ubiquitin ligase acting in the mitotic pathway differed from that of the S-phase pathway, we decided to test whether the type of ubiquitin chains built on $\mathrm{Mcm} 7$ in mitosis also differed. To determine which ubiquitin chains were required for the mitotic Mcm7 ubiquitylation and replisome disassembly, we supplemented extract with Cullin inhibitor, allowed completion of DNA synthesis and subsequently induced mitosis along with addition of a series of ubiquitin mutants that have only one lysine left in their sequence (Fig 1G). We observed that only wt ubiquitin and ubiquitin containing lysine 6 (K6) or lysine 63 (K63) could support mitotic replisome disassembly (as visualised by absence of Cdc45 on chromatin at 75 min after inducing mitosis) (Fig 1G). Interestingly, chains linked through lysine 48 (K48), which are responsible for S-phase unloading (Moreno et al., 2014), could still be attached to Mcm7 in mitosis (upshift of modified Mcm7 forms) but they could not support unloading of the replisome as Cdc45 remained associated with chromatin. In a reciprocal experiment, we used a series of ubiquitin mutants with only one of the lysines within ubiquitin mutated (Fig $1 \mathrm{H})$. All of the mutants used, apart from the UbiNOK control mutant, supported disassembly 
1 of the replisome, suggesting that either $\mathrm{K} 6$ or $\mathrm{K} 63$ can fulfill the mitotic pathway

2 requirements $($ Fig $1 \mathrm{H})$.

Having established that the type of ubiquitin chains and the type of ubiquitin ligase used by the mitotic pathway were different to those used by the S-phase pathway, our aim was to determine which ligase is driving this mitotic disassembly pathway. To identify the potential ligase we decided to immunoprecipitate the replisome retained on mitotic chromatin and analyse all the interacting proteins by mass spectrometry. We set up a replication reaction in the presence of caffeine and the p97 inhibitor and induced mitosis upon completion of DNA synthesis. We immunoprecipitated Mcm3 from mitotic chromatin and analysed interacting factors by mass spectrometry. Firstly, we determined which components of the replisome are still retained on chromatin in mitosis. For this we compared the replisome components retained on chromatin in mitosis with the S-phase post-termination replisome, reported previously (Sonneville et al., 2017) (Fig 2A and B). Interestingly, while inhibition of replisome disassembly in S-phase led to accumulation of the whole replisome on chromatin (Sonneville et al., 2017), only a selection of replisome components stayed on chromatin in mitosis. All of the lagging strand components of the replisome were lost, as were Mcm10 and Claspin, while levels of Ctf4/And-1, Timeless/Tipin and Pol epsilon were also reduced (Fig $2 \mathrm{~A}$ and $\mathrm{B}$ ). This suggests that only components directly interacting with the $\mathrm{CMG}$ accumulated around it through to mitosis, while others, more peripheral to CMG could dissociate over time.

The level of histone chaperone FACT (Spt16 and SSRP) stays the same between Sphase and mitosis. This suggests that the retained replisome has the potential ability to move through chromatin as FACT is likely to displace nucleosomes in front of such a replisome. We could see also that Cul2 ${ }^{\mathrm{LRR} 1}$, which strongly accumulated in the S-phase post-termination replisome, is not a major component of the mitotic replisome, as expected from previous data (Fig 1).

Finally, we detected two other ubiquitin ligases interacting with the mitotic helicase: TRAIP and RNF213. More specifically, we found that TRAIP interacts with the posttermination replisome in S-phase but it is enriched in mitosis, while RNF213 is a minor interactor of only the mitotic replisome (Fig 2A). The TNF-receptor-associated factor (TRAF)interacting protein (TRAIP, also known as TRIP or RNF206) was originally identified through its ability to bind TRAF1 and TRAF2 and shown to inhibit NFkB activation (Lee, Lee et al., 1997). It has been since shown that TRAIP is an E3 ubiquitin ligase, which is essential for cell proliferation (Besse, Campos et al., 2007, Park, Choi et al., 2007), and which is required for resolution of replication stress (Feng, Guo et al., 2016, Harley, Murina et al., 2016, Hoffmann, Smedegaard et al., 2016) and for regulation of the spindle assembly checkpoint during mitosis (Chapard, Meraldi et al., 2014). TRAIP is ubiquitously expressed, with its 
1 expression regulated by E2F transcription factors and protein stability controlled by the ubiquitin proteasome pathway - as a result, the protein level of TRAIP peaks in the G2/M stage of the cell cycle (Chapard, Hohl et al., 2015). On the other hand, RNF213 (mysterin) is a large $(591 \mathrm{kD})$ ATPase/E3 ligase, which is mostly known as being a susceptibility gene for Moyamoya disease (MMD) (cerebrovascular disease) (Kamada, Aoki et al., 2011, Liu, Morito et al., 2011). Of note, RNF213-/- mice do not show any apparent health problems (Kobayashi, Yamazaki et al., 2013, Sonobe, Fujimura et al., 2014) and more recently RNF213 was shown to globally regulate ( $\alpha$-ketoglutarate)-dependent dioxygenases ( $\alpha$ KGDDs) and non-mitochondrial oxygen consumption (NMOC) (Banh, lorio et al., 2016). To support our mass spectrometry data, we have tested a number of antibodies by western blotting against RNF213 and TRAIP to confirm their association with the chromatin bound replisome in mitosis. Whilst we were unsuccessful with detection of any signal for RNF213, we were able to show that TRAIP interacts with replisome retained on chromatin in mitosis (Fig 2C and EV Fig1D).

As we confirmed that TRAIP is a likely candidate for the ubiquitin ligase ubiquitylating Mcm7 and leading to disassembly of the replisome retained in mitosis, we next characterized TRAIP chromatin binding dynamics during the two cell cycle stages and the replisome disassembly process. TRAIP associated weakly with S-phase chromatin at times when forks are moving through chromatin replicating DNA. However, it accumulated strongly on chromatin in S-phase upon inhibition of replisome disassembly with the p97 inhibitor (Fig 3A). Importantly, TRAIP also accumulated on mitotic chromatin when replisome disassembly was inhibited with the p97 inhibitor, following the same pattern as replisome components (Fig 3 B). To test whether TRAIP is the ubiquitin ligase ubiquitylating Mcm7 in mitosis, we purified recombinant GST-tagged X.laevis TRAIP, both wild type and C25A RING domain mutant, which been shown to disrupt TRAIP ubiquitin ligase activity (Besse et al., 2007, Chapard et al., 2014). We blocked the disassembly of the replisome in S-phase by inhibition of cullins with MLN4924, and drove extract into mitosis by addition of Cyclin $\mathrm{A} 1 \Delta$, when we added recombinant wt or mutant TRAIP. To be able to clearly see ubiquitylation of Mcm7 in mitosis we supplemented the mitotic extract also with p97 inhibitor NMS873 to inhibit unloading of ubiquitylated Mcm7. Figure $3 \mathrm{C}$ shows that addition of RING mutant of TRAIP, but not wild type TRAIP, inhibits mitotic ubiquitylation of Mcm7 as the size of ubiquitylated forms of Mcm7 remains very close to ones build on Mcm7 already in S-phase (15 min timepoint). This suggest that the recombinant mutant TRAIP successfully competed with endogenous TRAIP protein and that ubiquitin ligase activity of TRAIP is needed for mitotic Mcm7 ubiquitylation.

To fully understand the requirement for ubiquitin like modifications during the mitotic replisome disassembly in vertebrates, we aimed to establish whether SUMOylation plays 
1 any role in this process as ULP-4 is essential for mitotic helicase disassembly in C. elegans embryos. To this end we decided to inhibit or stimulate SUMOylation during mitosis and assess its effect on replisome disassembly. Firstly, we observed that late S-phase chromatin is full of SUMOylated factors and that levels of these proteins go down over time upon entry into mitosis (Fig 4 and EV Fig 2). To inhibit SUMOylation we have supplemented the mitotic extract with the recombinant active domain of SENP1 which acts as a potent non-specific deSUMOylating enzyme. Addition of SENP1 wipes out all the SUMO2ylation (Fig 4A) and SUMO1ylation (EV Fig 3A) but the disassembly of the mitotic replisome is not affected (Fig 4A). We have also stimulated SUMOylation through addition of a high concentration of recombinant SUMO1 or SUMO2 (EV Fig 2). In both cases, despite a clear increase of SUMO signal on chromatin, the unloading of the mitotic replisome was not affected. Finally we have also blocked de-SUMOylation with SUMO2-VS, a derivative of SUMO2 which binds to the active site of SENPs and blocks their activity. Again, we could observe strong accumulation of SUMO2ylated products in the extract and on chromatin without affecting mitotic replisome disassembly. Interestingly, despite inhibition of de-SUMOylating enzymes, most of the SUMO signal is still disappearing from chromatin during progression of mitosis, indicating that the SUMOylated proteins are unloaded from chromatin throughout mitosis rather than being de-SUMOylated. In conclusion, we determined that SUMO modifications do not play an essential role in the mitotic disassembly pathway in Xenopus egg extract. In an analogous way we have also shown that they do not play a role during the S-phase replisome disassembly pathway (EV Fig 3).

Finally, we set out to determine whether the mitotic replisome disassembly pathway we were characterising was a mere "back-up" pathway for replisomes that terminated in Sphase but failed to be unloaded, or if it can have a more generic usage to remove any replication machinery still remaining on chromatin until mitosis. To test such a possibility, we blocked active (stalled) replisomes on chromatin by addition of polymerase inhibitor aphidicolin to the egg extract during DNA replication reaction. To accumulate such replisomes in large numbers, we also supplemented the extract with caffeine so as to block checkpoint activation and fire origins uncontrollably. Upon accumulation of such blocked replisomes we supplemented the reaction optionally with Cyclin $\mathrm{A} 1 \Delta$ at 90 min to induce mitotic entry (Fig 5A). Interestingly, active replisomes remained associated with chromatin throughout the experiment in late S-phase (buffer control), with no indication of Mcm7 ubiquitylation as expected. Upon addition of cyclin A1 $\Delta$ however, Mcm7 becomes ubiquitylated and replisomes are unloaded (Cyclin A1 $\Delta$ ). We can observe however, a noticeable delay of both these processes compared with terminated replisomes (compare Fig $5 \mathrm{~A}$ with Fig 1B). Such a delay is likely due to the fact that with no prior ubiquitylation of Mcm7 in S-phase, it takes longer for ubiquitin chains to be built in mitosis. We have also 
1 determined that unloading of stalled replisomes requires the activity of p97 segregase, as it is inhibited with p97 inhibitor NMS873 (Fig 5B). From these observations we can thus say that neither prior modification of Mcm7 in S-phase nor the "terminated" conformation of the helicase are essential for mitotic modification of Mcm7 and replisome disassembly.

\section{DISCUSSION}

We have presented here the existence of a mitotic pathway of replisome disassembly in vertebrates. One immediate question is why would the cells need a mitotic pathway of replisome disassembly? Traditionally, it is perceived that all the DNA metabolism should be finished before cells enter mitosis. According to this model, G2 phase of the cell cycle is there to ensure that all DNA replication and damage repair are completed prior to chromosome condensation and separation during mitosis. The last decade provided, however, much evidence that this is not the case: unreplicated DNA is detected in many human cells in mitosis; DNA synthesis can proceed during mitosis (mitotic DNA synthesis MiDAS); underreplicated DNA can lead to the formation of ultrafine bridges (UFB) in anaphase and, finally, formation of structures in $\mathrm{G} 1$ stage of the next cell cycle that are bound by 53BP1 protein (53BP1 bodies) (Liu, Nielsen et al., 2014, Minocherhomji, Ying et al., 2015, Moreno, Carrington et al., 2016). Genomewide, such unreplicated regions correlate with common fragile sites (CFS), which are chromosomal loci reponsible for the majority of the rearrangements found in cancer cells (Bhowmick \& Hickson, 2017). These unreplicated fragments of DNA result from replication forks not finishing replication and such forks, with their associated replisomes, are subsequently retained on chromatin into mitosis. It is likely that these unreplicated DNA fragments must be processed in mitosis to ensure correct chromosome segregation and this processing will involve replisome unloading and fork remodelling - hence the need for a process of replisome disassembly in mitosis.

TRAIP is a pleiotropic ubiquitin ligase involved in numerous cellular processes. It is clear that TRAIP is essential for appropriate repair of DNA damage in many forms: mitomycin C (MMC) induced inter-strand crosslinks (ICLs); damage caused by treatments with campthotecin (CPT) (Hoffmann et al., 2016), UV (Harley et al., 2016) and hydroxyurea (HU) (Feng et al., 2016); as well as for translesion DNA synthesis (Wallace, Merkle et al., 2014). TRAIP has also been reported to be an important regulator of the spindle assembly checkpoint and regulates mitotic progression. Cells with downregulated TRAIP go through mitosis faster and with more chromosome segregation errors (Chapard et al., 2014, Park, Jo

36 et al., 2015). For most of these processes the ubiquitin ligase activity of TRAIP is essential, 37 but the substrate(s) modified by TRAIP is not known. 
In support of our observation that TRAIP interacts weakly with S-phase chromatin when replication forks replicate DNA (Fig 3A), TRAIP has been shown to interact with nascent DNA in unperturbed S-phase in human cells through Nascent Chromatin Capture (NCC) (Hoffmann et al., 2016) but TRAIP knockdown does not affect much replication progression and overall DNA synthesis rates (Harley et al., 2016, Hoffmann et al., 2016). Upon DNA damage TRAIP re-localises from nucleoli to sites of damage in a manner dependent on a PCNA interacting box (PIP-box), present at the C-terminus of TRAIP (Feng et al., 2016, Hoffmann et al., 2016). Loss of TRAIP was suggested to interfere with the reconfiguration of stalled replication forks (Hoffmann et al., 2016), possibly through unloading of PCNA as further inhibition of proteasomal degradation in the absence of TRAIP did not exacerbate the levels of $\mathrm{HU}$-induced fork stalling. This suggested that degradation of a TRAIP ubiquitylation substrate is not the cause of this phenotype (Feng et al., 2016, Hoffmann et al., 2016). Interestingly, cells expressing the $\triangle$ RING mutant of TRAIP as the only TRAIP version, are as sensitive to MMC as TRAIP knockdown cells, while $\triangle$ PIP TRAIP cells are only mildly sensitive. This indicates that even without PCNA interaction TRAIP can still find its targets at the replication forks (Hoffmann et al., 2016). With the data presented here, identifying TRAIP as the ubiquitin ligase needed for Mcm7 ubiquitylation during mitosis, it is interesting to speculate that TRAIP can play an analogous role during DNA damage repair i.e. to stimulate replisome unloading and fork remodeling.

If TRAIP interacts with replisome and post-termination replisome during normal S-phase (data presented here and (Hoffmann et al., 2016)) an important question emerges, which is how is it activated only in mitosis or only upon DNA damage? In human cells, the abundance of TRAIP protein is tightly regulated and peaks in G2/M stages of cell cycle (Chapard et al., 2015). Moreover, TRAIP re-localizes from nucleoli to sites of DNA damage upon treatment, but we do not know what is driving this change in location. In early mitosis TRAIP is localized all over nucleus but binds exclusively to chromosomes in anaphase (Chapard et al., 2014). The abundance, localization and activity of TRAIP are therefore very carefully regulated in the cell. The intricacy and importance of this regulation may stem from the fact that uncontrolled unloading of the replisome would have a big impact on cell viability and is supported by observations whereby even moderate levels of TRAIP overexpression were reported to be cytotoxic, while epitope tagging of TRAIP (especially at the N-terminus) affects its functionality (Hoffmann et al., 2016).

Our data is consistent with a model in which TRAIP drives mitotic replisome disassembly by promoting Mcm 7 modification with $\mathrm{K} 6$ and $\mathrm{K} 63$ linked ubiquitin chains. Although there is no previous experimental evidence that TRAIP can support such ubiquitin linkages in vivo, in vitro assays have shown that TRAIP works well with conjugating enzymes (E2s) 
1 UbcH5a,b,c (but not UbcH2, H3, H6, H7, or Ubc13+Uev1A) (Besse et al., 2007).

2 Interestingly, UbcH5a was shown to support formation of ubiquitin chains with no specific

3 topology (Windheim, Peggie et al., 2008). It is plausible therefore that TRAIP/UbcH5 can

4 effectively produce chains of different linkages to support mitotic replisome disassembly.

Upon Mcm7 ubiquitylation the replisome needs to be unloaded. In S-phase, K48 ubiquitin chains support replisome disassembly. In mitosis however, K48 chains are not functional and unloading is driven instead by $\mathrm{K} 6$ and $\mathrm{K} 63$ chains. We know that p97, in complex with Ufd1 and Npl4 cofactors, is responsible for unloading of the replisome in S-phase (Maric, Mukherjee et al., 2017, Moreno et al., 2014, Sonneville et al., 2017). While p97 is well known for processing substrates ubiquitylated with K48 linked ubiquitin chains (Meyer, Bug et al., 2012), less is known about its contribution in processing other ubiquitin linkages. Interestingly, a recent study shows that upon inhibition of p97 activity, human cells accumulate K6, K11, K48 and, to a lesser extent, K63 ubiquitin chains (Heidelberger, Voigt et al., 2018). Moreover, out of five tested p97 cofactors, all were found to associate with K11 chains, four with K48 chains, and three with K63 chains (Alexandru, Graumann et al., 2008). p97 cofactors are known also to interact with ubiquitin-like modifiers e.g. Nedd8 and Atg8 (reviewed in (Meyer, 2012)). Finally, p97 was also shown to bind more readily to branched K11-K48 chains, than to K11 or K48 chains on their own (Meyer \& Rape, 2014). These proteome-wide data imply that the role of p97 does indeed extend beyond recognition of K48-chain-modified substrates, though currently little is known about its interaction with K6 chains.

Importantly, other p97 substrates have been identified, which are modified with non-K48 chains e.g. in double stand break repair RNF8 multi-monoubiquitylates L3MBTL1, which is then extracted by p97 (Kato, Nakajima et al., 2014); in mitochondrial fusion, Mfn1 is ubiquitylated with K63 chains and processed by p97 (Mukherjee \& Chakrabarti, 2016) and in antiviral signaling the interaction between p97 and the RIG-I sensor of viral DNA is stimulated by K63 ubiquitylation of RIG-I (Hao, Jiao et al., 2015). All in all, there is much more complexity to $\mathrm{p} 97$ function regulation than generally appreciated and future research will reveal how the fine tuning of replisome disassembly by p97 at different stages of the cell cycle or from different DNA structures is achieved.

Finally, we have shown that in the Xenopus system neither S-phase nor mitotic replisome disassembly requires SUMO modifications (Fig 4 and EV Fig 3 ) in contrast to $C$. elegans embryos where ULP-4 is required for mitotic unloading (Sonneville et al., 2017). This requirement may be thus specific to worm embryos, require ULP-4 protein but not its enzymatic activity or regulate an indirect process that is not well recapitulated in the egg extract cell free system. It has been suggested recently that SUMOylation of TRAIP can 
1 regulate its stability and ability to move to the nucleus (Park, Han et al., 2016), but this may not be present in the egg extract.

Perturbations in DNA replication initiation and elongation leading to genomic instability are well linked with genetic disorders and can drive cancer development. The disruption of replisome disassembly is, therefore, highly likely to be detrimental to human health too. While so far we have no solid data to support this claim, previous studies with TRAIP do suggest this to be the case: homozygous TRAIP knockdown mouse embryos die shortly after implantation due to proliferation defects (Park et al., 2007); mutations in human TRAIP lead to primordial dwarfism (Harley et al., 2016); overexpression of human TRAIP has been reported in basal cell carcinomas (Almeida, Ryser et al., 2011) and breast cancer (Yang, Trent et al., 2006, Zhou \& Geahlen, 2009) and reduced nuclear expression of TRAIP was associated with human lung adenocarcinoma (Soo Lee, Jin Chung et al., 2016). The fact that cells have evolved multiple pathways to ensure timely replisome disassembly supports the notion of the vital importance of this process for cell biology and time will tell whether targeting Mcm7 and replisome disassembly in mitosis is the key mechanism leading to any of these disease phenotypes.

MATERIALS AND METHODS Inhibitors

Caffeine (C8960, Sigma) was dissolved in water at $100 \mathrm{mM}$ and added to the extract along with demembranated sperm nuclei at 5 mM. MLN4924 (A01139, Active Biochem) was dissolved in DMSO at $20 \mathrm{mM}$ and added to the extract 15 minutes after addition of sperm nuclei at $10 \mu \mathrm{M}$. NMS873 (17674, Cayman Chemical Company) was dissolved in DMSO at $10 \mathrm{mM}$ and added to the extract 15 minutes after addition of sperm nuclei at $50 \mu \mathrm{M}$. SUMO2-VS (UL-759) was purchased from Boston Biochem and used at $1 \mu \mathrm{M}$ in Xenopus laevis egg extract. Aphidicolin was dissolved in DMSO at $8 \mathrm{mM}$ and added to the extract along with demembranated sperm nuclei at $40 \mu \mathrm{M}$.

\section{Recombinant proteins}

Recombinant His-tagged ubiquitin and ubiquitin mutants were purchased from Boston Biochem, dissolved in LFB1/50 (40 mM Hepes/KOH pH 8.0, $20 \mathrm{mM}$ potassium phosphate $\mathrm{pH}$ 8.0, $50 \mathrm{mM}$ potassium chloride, $2 \mathrm{mM}$ magnesium chloride, $1 \mathrm{mM}$ EGTA; $10 \%$ sucrose $\mathrm{w} / \mathrm{v} ; 2 \mathrm{mM}$ DTT; $1 \mu \mathrm{g} / \mathrm{ml}$ aprotinin; $1 \mu \mathrm{g} / \mathrm{ml}$ leupeptin; $1 \mu \mathrm{g} / \mathrm{ml}$ pepstatin) buffer at $10 \mathrm{mg} / \mathrm{ml}$ and used at $0.5 \mathrm{mg} / \mathrm{ml}$ in Xenopus laevis egg extract. 
1 pET28a-X.I. SUMO1 and pET28a-X.I. SUMO2 were purchased from GenScript.

2 Recombinant His-tagged Xenopus laevis SUMO1 and SUMO2 were expressed in Rosetta

3 (DE3) pLysS cells over night at 20ㅇ after induction with $1 \mathrm{mM}$ IPTG. Cells were lysed in

4 lysis buffer: $50 \mathrm{mM}$ Tris- $\mathrm{HCl}, 500 \mathrm{mM} \mathrm{NaCl}, 10 \mathrm{mM}$ imidazole, $2 \mathrm{mM} \mathrm{MgCl}$, $0.1 \mathrm{mM} \mathrm{PMSF}$,

$51 \mu \mathrm{g} / \mathrm{ml}$ of each aprotinin, leupeptin and pepstatin, $\mathrm{pH} 7.5$. Homogenates were supplemented

6 with $25 \mathrm{U} / \mathrm{ml}$ benzonase and incubated at room temperature for 20 minutes. Homogenates

7 were subsequently spun down at $14,000 \mathrm{~g}$ for 30 minutes at $4^{\circ} \mathrm{C}$ and supernatants

8 incubated with $2 \mathrm{ml}$ of pre-washed Super Ni-NTA Affinity Resin (SUPER-NINA100,

9 Generon) for 2 hours with rotation at $4{ }^{\circ} \mathrm{C}$. Resins were subsequently washed twice with 50

$10 \mathrm{mM}$ Tris- $\mathrm{HCl}, 500 \mathrm{mM} \mathrm{NaCl}, 30 \mathrm{mM}$ imidazole, $0.1 \mathrm{mM}$ PMSF, $1 \mu \mathrm{g} / \mathrm{ml}$ of each aprotinin,

11 leupeptin and pepstatin, $\mathrm{pH}$ 7.5. Resin-bound proteins were finally eluted in $1 \mathrm{ml}$ fractions

12 with a solution containing $50 \mathrm{mM}$ Tris- $\mathrm{HCl}, 150 \mathrm{mM} \mathrm{NaCl}, 200 \mathrm{mM}$ imidazole, $5 \mathrm{mM} \beta$ -

13 mercaptoethanol, $0.1 \mathrm{mM} \mathrm{PMSF}, 1 \mu \mathrm{g} / \mathrm{ml}$ of each aprotinin, leupeptin and pepstatin, $\mathrm{pH}$ 7.5.

14 Fractions containing the highest levels of recombinant SUMO1 or SUMO2 were dialysed into

15 LFB1/50 buffer. Both SUMO1 and SUMO2 were used at $0.5 \mathrm{mg} / \mathrm{ml}$ in Xenopus laevis egg 16 extract.

17 pET28a-pHISTEV30a-SENP1(415-649) was a kind gift from Ron Hay's laboratory.

18 Recombinant active domain of human SENP1 (aa 415-647) was expressed and purified as 19 explained above for recombinant SUMOs.

20 Recombinant His-tagged Xenopus laevis cyclin A1 N $\Delta 56$ (pET23a-X.I. cyclin A1 N $\Delta 56$ ) was a kind gift from Julian Blow's laboratory (Strausfeld et al., 1996), was expressed in Rosetta (DE3) pLysS cells over night at 15을 after induction with $1 \mathrm{mM}$ IPTG, and subsequently purified as explained above for recombinant SUMOs but using different solutions. Lysis buffer: $50 \mathrm{mM}$ Tris- $\mathrm{HCl}, 300 \mathrm{mM} \mathrm{NaCl}, 2 \mathrm{mM} \mathrm{MgCl}_{2}, 1 \mathrm{mM}$ DTT, $0.1 \mathrm{mM} \mathrm{PMSF}, 1 \mu \mathrm{g} / \mathrm{ml}$ of each aprotinin, leupeptin and pepstatin, $\mathrm{pH}$ 7.4. Washes: Resin was washed twice with lysis buffer on its own and twice again with lysis buffer supplemented with $0.1 \%$ Triton X-100. Elution buffer: Lysis buffer supplemented with $10 \%$ glycerol and $250 \mathrm{mM}$ imidazole. Xenopus TRAIP was cloned into pGS21 vector, expressed in BL21 (DE3) bacterial strain in autoinduced AIM media O/N at 18으. Pellets were lysed in lysis buffer: $50 \mathrm{mM} \mathrm{NaH2PO4;} \mathrm{pH}$ 9, 300mM NaCl, 10\% glycerol, 2mM DTT, $2 \mathrm{mM} \mathrm{MgCl}_{2}, 0.05 \%$ Brij, $0.1 \mathrm{mM} \mathrm{PMSF,} 1 \mu \mathrm{g} / \mathrm{ml}$ of each aprotinin, leupeptin and pepstatin, $1 \mathrm{mg} / \mathrm{ml}$ lysozyme, $25 \mathrm{U} / \mathrm{ml}$ Benzonase. The protein was purified as above but using Glutathione Sepharose 4B (GE Healthcare) and eluted with $25 \mathrm{mM}$ glutathione. The protein was then dialised into LFB1/50 buffer (as above) and concentrated up to $0.3 \mathrm{mg} / \mathrm{ml}$ of full length GST-TRAIP. It was used in the egg extract at final concentration of $30 \mu \mathrm{g} / \mathrm{ml}$. pGS21-TRAIP(C25A) was generated by site directed mutagenesis and purified in analogous way. 
Antibodies

a-PCNA (P8825) and $\alpha-H I S$ (H1029) were purchased from Sigma. $\alpha$-TRAIP (NBP1-87125) and $\alpha-R N F 213$ (NBP1-88466) were purchased from Novus Biologicals. $\alpha-S U M O 2$ and $\alpha-$ SUMO1 were produced in the lab by culturing the hybridoma cell line SUMO2 (8A2) and SUMO1 (21C7) purchased from Developmental Studies Hybridoma Bank (hybridoma cell culture was done following manufacturer's instructions and adding $20 \mathrm{mM} \mathrm{L-glutamine}$ to the media). Affinity purified a-Cdc45 (Gambus, Khoudoli et al., 2011), a-Mcm3 (Khoudoli, Gillespie et al., 2008) and a-LRR1 (S962D) (Sonneville et al., 2017) were previously described. a-Mcm7 was raised in sheep against recombinant Xenopus laevis Mcm7, purified from E.coli and affinity purified in the lab.

\section{DNA staining and microscopy}

Interphase Xenopus laevis egg extract was supplemented with $10 \mathrm{ng} / \mu \mathrm{l}$ of demembranated sperm nuclei and incubated at $23^{\circ} \mathrm{C}$ for 90 minutes to allow completion of DNA replication. Mitosis was optionally driven by addition of $826 \mathrm{nM}$ cyclin $\mathrm{A} 1 \mathrm{~N} \Delta 56$, and reactions were incubated for further 2 hours. Assembled S-phase or mitotic chromatin were stained with Hoechst 33258 and viewed as previously described (Strausfeld et al., 1996).

\section{DNA synthesis assay (TCA)}

The replication reactions were started with the addition of demembranated Xenopus sperm DNA to $10 \mathrm{ng} / \mu \mathrm{l}$ as described before (Gillespie et al., 2012). The synthesis of nascent DNA was measured by quantification of a32P-dATP incorporation into newly synthetized DNA as described before (Gillespie et al., 2012).

\section{Chromatin isolation time-course}

Interphase Xenopus laevis egg extract was supplemented with $10 \mathrm{ng} / \mu \mathrm{l}$ of demembranated sperm DNA and subjected to indicated treatments. The reaction was incubated at $23^{\circ} \mathrm{C}$ for 90 minutes to allow completion of DNA replication, after which mitosis was driven by addition of $826 \mathrm{nM}$ cyclin $\mathrm{A} 1 \mathrm{~N} \Delta 56$. The extract was optionally supplemented with inhibitors or recombinant proteins along with cyclin $\mathrm{A} 1 \mathrm{~N} \Delta 56$ as indicated. Mitotic chromatin was isolated in ANIB100 buffer supplemented with $10 \mathrm{mM}$ 2-chloroacetamide (Millipore) and $5 \mathrm{mM} \mathrm{N}$ ethlylmaleimide (NEM) (Acros Organics) at indicated time-points after addition of cyclin A1 $\mathrm{N} \Delta 56$ as previously described (Gillespie et al., 2012). 
$13.75 \mathrm{ml}$ of interphase Xenopus laevis egg extract was supplemented with $10 \mathrm{ng} / \mathrm{\mu l}$ of

2 demembranated sperm nuclei, $5 \mathrm{mM}$ caffeine and $50 \mu \mathrm{M}$ p97 inhibitor NMS873. The

3 reaction was incubated at $2^{\circ} \mathrm{C}$ for 90 minutes to allow completion of DNA replication, after

4 which mitosis was driven by addition of recombinant cyclin A1 N $\Delta 56$ at $826 \mathrm{nM}$ followed by incubation at $23^{\circ} \mathrm{C}$ for further 60 minutes. At this stage, chromatin was isolated in ANIB100 buffer supplemented with $10 \mathrm{mM}$ 2-chloroacetamide as previously described (Gambus et al., 2011) and chromatin bound protein complexes released into solution by chromosomal DNA digestion with $2 \mathrm{U} / \mu \mathrm{l}$ benzonase for 15 minutes. Solubilisation of chromatin bound protein complexes was further facilitated by subjecting the sample to 5 minutes of 30 seconds ON/OFF sonication cycles using a diagenode bioruptor and increasing the concentration of potassium acetate up to $150 \mathrm{mM}$. The resulting protein complexes were subsequently subjected to either non-specific IgG (from sheep serum) or Mcm3 immunoprecipitation and the immunoprecipitated material analysed by mass spectrometry as previously described (Sonneville et al., 2017) in collaboration with Dr Richard Jones from MS Bioworks LLC.

\section{ACKNOWLEDGMENTS}

Dr Sara Priego Moreno was funded by Wellcome Trust ISS Award, Dr Rebecca Jones and Dr Agnieszka Gambus were funded by MRC CDA MR/K007106/1. We would like to thank Prof Ron Hay for pET28a-pHISTEV30a-SENP1(415-649) and Prof Julian Blow for pET23a-X.I. cyclin A1 N $\Delta 56$.

\section{FIGURE LEGENDS}

Figure 1. Mcm7 is ubiquitylated with $\mathrm{K} 6$ and $\mathrm{K} 63$ ubiquitin chains in mitosis and removed from chromatin by $\mathrm{p} 97$ segregase. (A) Experimental design for driving egg extract into mitosis. (B) Experiment following design in (A). DNA was replicated to completion in egg extract supplemented with Cullin inhibitor MLN4924. After 90 min of replication reaction Cyclin A1 $\Delta$ was optionally added to the extract to drive extract to mitosis. Chromatin was isolated at indicated time-points after Cyclin A1 $\Delta$ addition and chromatin samples analysed by western blotting with indicated antibodies. Colloidal Coomassie stained histones serve as a quality and loading control. (C) The replication reaction was completed in the presence of

32 Cullin inhibitor MLN4924 and driven into mitosis by addition of Cyclin A1 $\Delta$. At the same time as Cyclin $A 1 \Delta$, half of the sample was supplemented additionally with p97 inhibitor NMS873. Chromatin samples were isolated at indicated time-points and analysed as in (B). A sample without DNA addition (no DNA) was processed alongside others as a chromatin specificity control. (D) The replication reaction was completed in the presence of p97 inhibitor NMS873 
1 and driven into mitosis by addition of Cyclin A1 $\Delta$. At the same time as Cyclin $A 1 \Delta$, samples were supplemented with recombinant wt ubiquitin or UbiNOK. Chromatin samples were analysed as above. (E) Experiment as in (D) but replication reaction was carried out in the presence of Cullin inhibitor MLN4924 instead of p97 inhibitor. (G) and (H) Replication reaction was completed in the presence of Cullin inhibitor MLN4924 and driven to mitosis by addition of Cyclin $\mathrm{A} 1 \Delta$. At the same time as Cyclin $\mathrm{A} 1 \Delta$ addition, extract was supplemented with indicated mutants of ubiquitin. Chromatin was isolated at 75 minutes after Cyclin A1 $\Delta$ addition and analysed by western blotting as above.

Figure 2. Composition of the replisome retained on mitotic chromatin. (A) Replication reaction was completed in egg extract supplemented with caffeine and p97 inhibitor NMS873, extract was driven into mitosis by addition of Cyclin $A 1 \Delta$, chromatin isolated at 60 minutes after Cyclin $\mathrm{A} 1 \Delta$ addition and chromatin proteins released from DNA. Antibodies against Mcm3 (or control lgG) were used to immunoprecipitate replisomes and immunoprecipitated samples were analysed by mass spectrometry. The total spectral count for each identified replisome component is presented together with sequence coverage of analysed peptides. The result of analysis of mitotic retained replisome is compared with Sphase post-replication replisome reported in (Sonneville et al., 2017). (B) Schematic representation of the data presented in (A). (C) A small proportion of the material from the mitotic Mcm3 IP experiment in (A) was analysed by western blotting with indicated antibodies.

Figure 3. TRAIP regulates replisome disassembly in mitosis. (A) Sperm DNA was replicated in egg extract optionally supplemented with p97 inhibitor NMS873. Chromatin samples were isolated during reaction at indicated time-points and analysed as in Fig 1. (B) Experiment analogous to Fig $1 \mathrm{C}$ but analysed with indicated antibodies. (C) The replication reaction was completed in the presence of Cullin inhibitor MLN4924 and driven into mitosis by addition of Cyclin $\mathrm{A} 1 \Delta$. At the same time as Cyclin $\mathrm{A} 1 \Delta$, most of the samples were supplemented with p97 inhibitor NMS873 and optionally with LFB1/50 buffer or wt TRAIP or RING mutant (C25A) TRAIP at $30 \mu \mathrm{g} / \mathrm{ml}$. Chromatin samples were isolated at indicated timepoints and analysed with indicated antibodies. The dashed line on $\mathrm{Mcm} 7 \mathrm{blot}$ runs through the middle of ubiquitylation signal for Mcm7 in mitosis in control (buffer) sample to aid comparison between samples.

Figure 4. SUMOylation is not required for mitotic replisome disassembly. (A) The replication reaction was completed in the presence of Cullin inhibitor MLN4924 and driven 
1 into mitosis by addition of Cyclin $\mathrm{A} 1 \Delta$. At the same time as Cyclin $\mathrm{A} 1 \Delta$, half of the sample

2 was supplemented additionally with active domain of SENP1. Chromatin samples were

3 isolated at indicated time-points and analysed as in Fig 1. (B) As in (A) but instead of

4 supplementing extract with SENP1 we supplemented it with SENPs inhibitor SUMO2-VS.

Figure 5. Mitotic unloading of active helicases. (A) The replication reaction was performed in egg extract supplemented with DNA polymerase inhibitor aphidicolin and checkpoint inhibitor caffeine. After 90 min of reaction, Cyclin A1 $\Delta$ was optionally added and chromatin samples isolated at indicated time-points and analysed by western blotting with indicated antibodies (analogously to Fig 1B). (B) The inhibition of stalled replisomes was achieved as in (A) and extract was driven into mitosis with optional supplementation with p97 inhibitor NMS873. Chromatin samples were analysed as in (A).

EV Figure 1. Setting up mitotic replisome disassembly. (A) Progression of replication reaction was measured by incorporation of radioactive dATP into the DNA. $P^{32} \alpha-d A T P$ was added to the extract at the beginning of the reaction (S-phase) or after $90 \mathrm{~min}$ of replication reaction together with optional addition of Cyclin $\mathrm{A} 1 \Delta$ (buffer control or Cyclin A1 $1 \Delta$ ). (B) The replication reaction was completed and optionally driven into mitosis by addition of Cyclin A1 $\Delta$. The nuclei assembled in the extract were visualised by Hoechst 33258 staining. (C) Experiment as in Fig $1 \mathrm{C}$ but indicated inhibitors were present in the reaction throughout both stages of the cell cycle.

EV Figure 2. Mitotic replisome disassembly is not affected by stimulation of

SUMOylation. The replication reaction was completed in the presence of Cullin inhibitor MLN4924 and driven into mitosis by addition of Cyclin A1 $\Delta$. At the same time as Cyclin A1 $\Delta$, extract was optionally supplemented with buffer control, SUMO1 or SUMO2. Chromatin samples were isolated at indicated time-points and analysed as in Fig 1.

EV Figure 3. S-phase replisome disassembly is not affected by SUMOylation. (A) The replication reaction was performed in egg extract supplemented with dominant negative mutant of Ubc9 (Ubc9DN) or active domain of SENP1. Chromatin samples were isolated at indicated time-points and analysed by western blotting with indicated antibodies. (B) The replication reaction was performed in egg extract supplemented with $0.5 \mathrm{mg} / \mathrm{ml}$ of SUMO1 or SUMO2 (as indicated). Chromatin samples were isolated at indicated time-points and analysed by western blotting with indicated antibodies. Controls as in Fig 1. 


\section{REFERENCES}

Alexandru G, Graumann J, Smith GT, Kolawa NJ, Fang R, Deshaies RJ (2008) UBXD7 binds multiple ubiquitin ligases and implicates p97 in HIF1alpha turnover. Cell 134: 804-16 Almeida S, Ryser S, Obarzanek-Fojt M, Hohl D, Huber M (2011) The TRAF-interacting protein (TRIP) is a regulator of keratinocyte proliferation. J Invest Dermatol 131: 349-57 Banh RS, Iorio C, Marcotte R, Xu Y, Cojocari D, Rahman AA, Pawling J, Zhang W, Sinha A, Rose CM, Isasa M, Zhang S, Wu R, Virtanen C, Hitomi T, Habu T, Sidhu SS, Koizumi A, Wilkins SE, Kislinger T et al. (2016) PTP1B controls non-mitochondrial oxygen consumption by regulating RNF213 to promote tumour survival during hypoxia. Nat Cell Biol 18: 803-813

Besse A, Campos AD, Webster WK, Darnay BG (2007) TRAF-interacting protein (TRIP) is a RING-dependent ubiquitin ligase. Biochem Biophys Res Commun 359: 660-4 Bhowmick R, Hickson ID (2017) The "enemies within": regions of the genome that are inherently difficult to replicate. F1000Res 6: 666 Burrell RA, McClelland SE, Endesfelder D, Groth P, Weller MC, Shaikh N, Domingo E, Kanu N, Dewhurst SM, Gronroos E, Chew SK, Rowan AJ, Schenk A, Sheffer M, Howell M, Kschischo M, Behrens A, Helleday T, Bartek J, Tomlinson IP et al. (2013) Replication stress links structural and numerical cancer chromosomal instability. Nature 494: 492-6 Chapard C, Hohl D, Huber M (2015) The TRAF-interacting protein (TRAIP) is a novel E2F target with peak expression in mitosis. Oncotarget 6: 20933-45 Chapard C, Meraldi P, Gleich T, Bachmann D, Hohl D, Huber M (2014) TRAIP is a regulator of the spindle assembly checkpoint. J Cell Sci 127: 5149-56 Dewar JM, Budzowska M, Walter JC (2015) The mechanism of DNA replication termination in vertebrates. Nature 525: 345-50

Feng W, Guo Y, Huang J, Deng Y, Zang J, Huen MS (2016) TRAIP regulates replication fork recovery and progression via PCNA. Cell Discov 2: 16016 Fu YV, Yardimci H, Long DT, Ho TV, Guainazzi A, Bermudez VP, Hurwitz J, van Oijen A, Scharer OD, Walter JC (2011) Selective bypass of a lagging strand roadblock by the eukaryotic replicative DNA helicase. Cell 146: 931-41

Gambus A, Jones RC, Sanchez-Diaz A, Kanemaki M, van Deursen F, Edmondson RD, Labib K (2006) GINS maintains association of Cdc 45 with MCM in replisome progression complexes at eukaryotic DNA replication forks. Nat Cell Biol 8: 358-66 Gambus A, Khoudoli GA, Jones RC, Blow JJ (2011) MCM2-7 form double hexamers at licensed origins in Xenopus egg extract. The Journal of biological chemistry 286: 11855-64 Gillespie PJ, Gambus A, Blow JJ (2012) Preparation and use of Xenopus egg extracts to study DNA replication and chromatin associated proteins. Methods 57: 203-13 Hao Q, Jiao S, Shi Z, Li C, Meng X, Zhang Z, Wang Y, Song X, Wang W, Zhang R, Zhao Y, Wong CC, Zhou Z (2015) A non-canonical role of the p97 complex in RIG-I antiviral signaling. EMBO J 34: 2903-20

Harley ME, Murina O, Leitch A, Higgs MR, Bicknell LS, Yigit G, Blackford AN, Zlatanou A, Mackenzie KJ, Reddy K, Halachev M, McGlasson S, Reijns MAM, Fluteau A, Martin CA, Sabbioneda S, Elcioglu NH, Altmuller J, Thiele H, Greenhalgh L et al. (2016) TRAIP promotes DNA damage response during genome replication and is mutated in primordial dwarfism. Nat Genet 48: 36-43

Heidelberger JB, Voigt A, Borisova ME, Petrosino G, Ruf S, Wagner SA, Beli P (2018) Proteomic profiling of VCP substrates links VCP to K6-linked ubiquitylation and c-Myc function. EMBO Rep 19

Hoffmann S, Smedegaard S, Nakamura K, Mortuza GB, Raschle M, Ibanez de Opakua A, Oka Y, Feng Y, Blanco FJ, Mann M, Montoya G, Groth A, Bekker-Jensen S, Mailand N 
(2016) TRAIP is a PCNA-binding ubiquitin ligase that protects genome stability after replication stress. J Cell Biol 212: 63-75

Kamada F, Aoki Y, Narisawa A, Abe Y, Komatsuzaki S, Kikuchi A, Kanno J, Niihori T, Ono M, Ishii N, Owada Y, Fujimura M, Mashimo Y, Suzuki Y, Hata A, Tsuchiya S, Tominaga T, Matsubara Y, Kure S (2011) A genome-wide association study identifies RNF213 as the first Moyamoya disease gene. J Hum Genet 56: 34-40

Kato K, Nakajima K, Ui A, Muto-Terao Y, Ogiwara H, Nakada S (2014) Fine-tuning of DNA damage-dependent ubiquitination by OTUB2 supports the DNA repair pathway choice. Mol Cell 53: 617-30

Khoudoli GA, Gillespie PJ, Stewart G, Andersen JS, Swedlow JR, Blow JJ (2008) Temporal profiling of the chromatin proteome reveals system-wide responses to replication inhibition. Current biology : CB 18: 838-43

Kobayashi H, Yamazaki S, Takashima S, Liu W, Okuda H, Yan J, Fujii Y, Hitomi T, Harada KH, Habu T, Koizumi A (2013) Ablation of Rnf213 retards progression of diabetes in the Akita mouse. Biochem Biophys Res Commun 432: 519-25

Lee SY, Lee SY, Choi Y (1997) TRAF-interacting protein (TRIP): a novel component of the tumor necrosis factor receptor (TNFR)- and CD30-TRAF signaling complexes that inhibits TRAF2-mediated NF-kappaB activation. J Exp Med 185: 1275-85

Liu W, Morito D, Takashima S, Mineharu Y, Kobayashi H, Hitomi T, Hashikata H, Matsuura N, Yamazaki S, Toyoda A, Kikuta K, Takagi Y, Harada KH, Fujiyama A, Herzig R, Krischek B, Zou L, Kim JE, Kitakaze M, Miyamoto S et al. (2011) Identification of RNF213 as a susceptibility gene for moyamoya disease and its possible role in vascular development. PLoS One 6: e22542

Liu Y, Nielsen CF, Yao Q, Hickson ID (2014) The origins and processing of ultra fine anaphase DNA bridges. Curr Opin Genet Dev 26: 1-5

Maric M, Maculins T, De Piccoli G, Labib K (2014) Cdc48 and a ubiquitin ligase drive disassembly of the CMG helicase at the end of DNA replication. Science 346: 1253596 Maric M, Mukherjee P, Tatham MH, Hay R, Labib K (2017) Ufd1-Npl4 Recruit Cdc48 for Disassembly of Ubiquitylated CMG Helicase at the End of Chromosome Replication. Cell Rep 18: 3033-3042

Meyer H (2012) p97 complexes as signal integration hubs. BMC Biol 10: 48 Meyer H, Bug M, Bremer S (2012) Emerging functions of the VCP/p97 AAA-ATPase in the ubiquitin system. Nature cell biology 14: 117-23

Meyer HJ, Rape M (2014) Enhanced protein degradation by branched ubiquitin chains. Cell 157: 910-21

Minocherhomji S, Ying S, Bjerregaard VA, Bursomanno S, Aleliunaite A, Wu W, Mankouri HW, Shen H, Liu Y, Hickson ID (2015) Replication stress activates DNA repair synthesis in mitosis. Nature 528: 286-90

Moreno A, Carrington JT, Albergante L, Al Mamun M, Haagensen EJ, Komseli ES, Gorgoulis VG, Newman TJ, Blow JJ (2016) Unreplicated DNA remaining from unperturbed $\mathrm{S}$ phases passes through mitosis for resolution in daughter cells. Proceedings of the National Academy of Sciences of the United States of America 113: E5757-64 Moreno SP, Bailey R, Campion N, Herron S, Gambus A (2014) Polyubiquitylation drives replisome disassembly at the termination of DNA replication. Science 346: 477-81 Moyer SE, Lewis PW, Botchan MR (2006) Isolation of the Cdc45/Mcm2-7/GINS (CMG) complex, a candidate for the eukaryotic DNA replication fork helicase. Proceedings of the National Academy of Sciences of the United States of America 103: 10236-41

Mukherjee R, Chakrabarti O (2016) Regulation of Mitofusin1 by Mahogunin Ring Finger-1 and the proteasome modulates mitochondrial fusion. Biochim Biophys Acta 1863: 3065-3083 
1 Park ES, Choi S, Kim JM, Jeong Y, Choe J, Park CS, Choi Y, Rho J (2007) Early embryonic

2 lethality caused by targeted disruption of the TRAF-interacting protein (TRIP) gene.

3 Biochem Biophys Res Commun 363: 971-7

4 Park IS, Han Y, Chung HJ, Jung YW, Kim Y, Kim H (2016) SUMOylation regulates nuclear

5 localization and stability of TRAIP/RNF206. Biochem Biophys Res Commun 470: 881-7

6 Park IS, Jo KS, Won HS, Kim H (2015) Dimerization of TRAF-interacting protein (TRAIP)

7 regulates the mitotic progression. Biochem Biophys Res Commun 463: 864-9

8 Sonneville R, Moreno SP, Knebel A, Johnson C, Hastie CJ, Gartner A, Gambus A, Labib K

9 (2017) CUL-2LRR-1 and UBXN-3 drive replisome disassembly during DNA replication

10 termination and mitosis. Nat Cell Biol

11 Sonobe S, Fujimura M, Niizuma K, Nishijima Y, Ito A, Shimizu H, Kikuchi A, Arai-Ichinoi

12 N, Kure S, Tominaga T (2014) Temporal profile of the vascular anatomy evaluated by 9.4-T

13 magnetic resonance angiography and histopathological analysis in mice lacking RNF213: a

14 susceptibility gene for moyamoya disease. Brain Res 1552: 64-71

15 Soo Lee N, Jin Chung H, Kim HJ, Yun Lee S, Ji JH, Seo Y, Hun Han S, Choi M, Yun M,

16 Lee SG, Myung K, Kim Y, Chul Kang H, Kim H (2016) TRAIP/RNF206 is required for

17 recruitment of RAP80 to sites of DNA damage. Nat Commun 7: 10463

18 Strausfeld UP, Howell M, Descombes P, Chevalier S, Rempel RE, Adamczewski J, Maller

19 JL, Hunt T, Blow JJ (1996) Both cyclin A and cyclin E have S-phase promoting (SPF)

20 activity in Xenopus egg extracts. J Cell Sci 109 ( Pt 6): 1555-63

21 Wallace HA, Merkle JA, Yu MC, Berg TG, Lee E, Bosco G, Lee LA (2014) TRIP/NOPO E3

22 ubiquitin ligase promotes ubiquitylation of DNA polymerase eta. Development 141: 1332-41

23 Windheim M, Peggie M, Cohen P (2008) Two different classes of E2 ubiquitin-conjugating enzymes are required for the mono-ubiquitination of proteins and elongation by polyubiquitin chains with a specific topology. Biochem J 409: 723-9

Yang C, Trent S, Ionescu-Tiba V, Lan L, Shioda T, Sgroi D, Schmidt EV (2006)

Identification of cyclin D1- and estrogen-regulated genes contributing to breast carcinogenesis and progression. Cancer Res 66: 11649-58

Zhou Q, Geahlen RL (2009) The protein-tyrosine kinase Syk interacts with TRAF-interacting protein TRIP in breast epithelial cells. Oncogene 28: 1348-56 


\section{Priego Moreno et al. Fig 1}

A

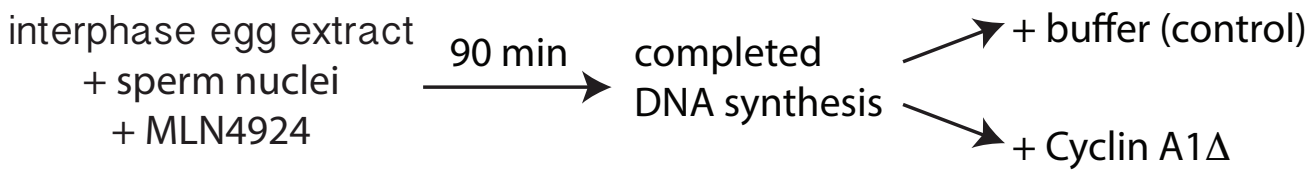

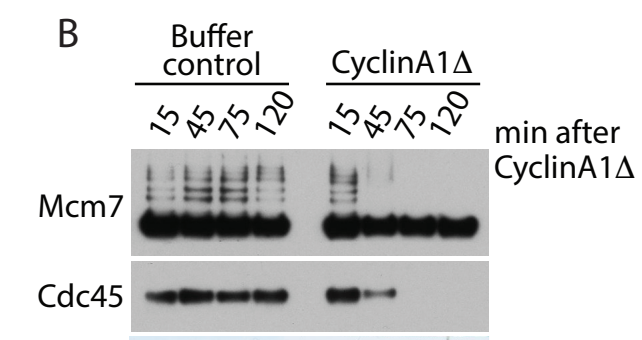

Histones

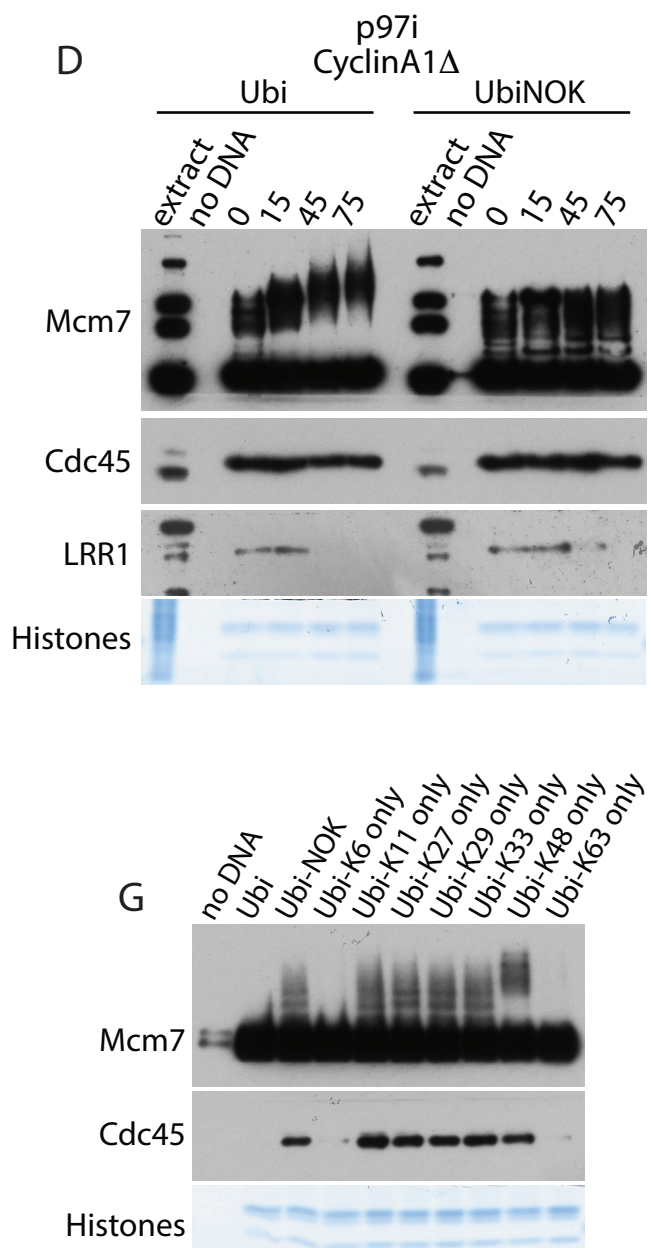

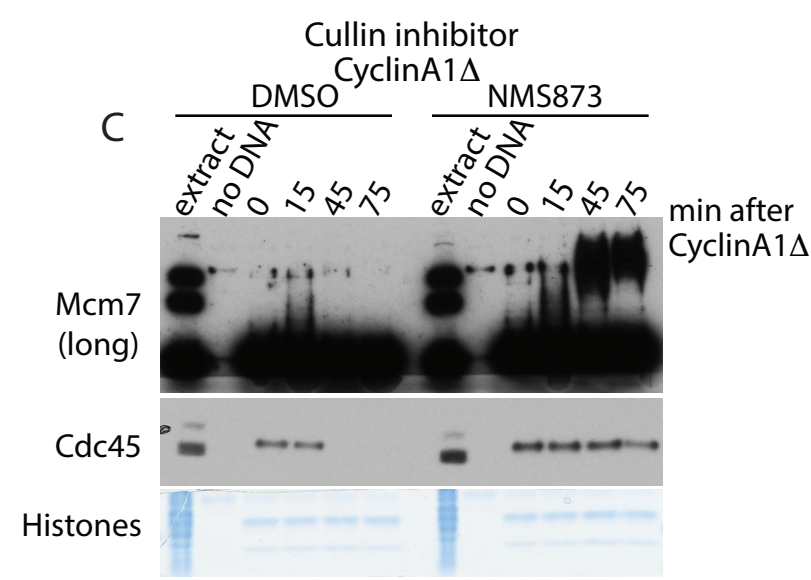
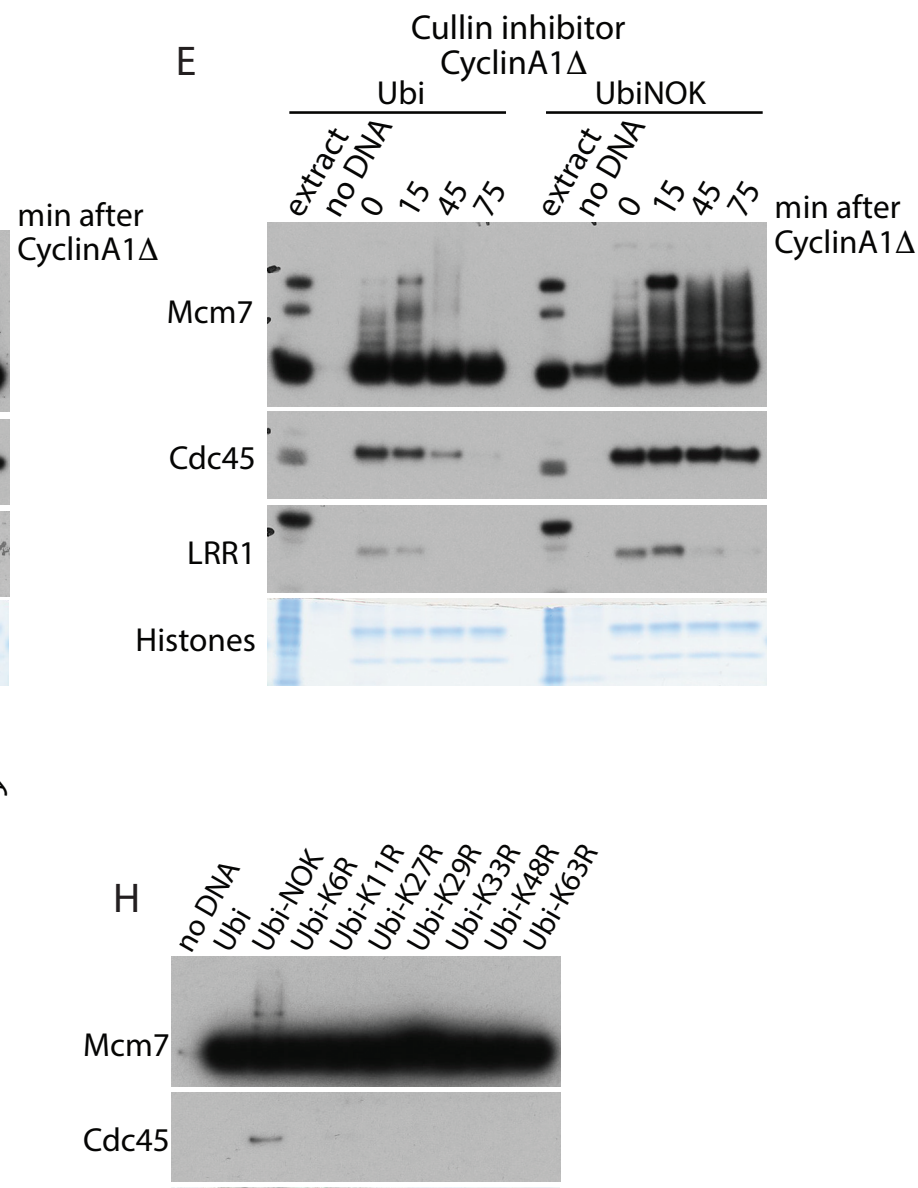

Histones 
Priego Moreno et al. Fig 2

\begin{tabular}{|c|c|c|c|c|c|}
\hline \multirow[t]{2}{*}{$A$} & & \multicolumn{2}{|c|}{$\begin{array}{l}\text { IP from mitotic chromatin } \\
\text { with retained CMG (extract } \\
\text { treated with p97i) }\end{array}$} & \multicolumn{2}{|c|}{$\begin{array}{l}\text { IP from S-phase post-termination } \\
\text { chromatin with retained } \\
\text { replisome (Sonneville et al 2017) }\end{array}$} \\
\hline & Protein (kDa) & $\begin{array}{c}\lg \mathrm{G} \\
\text { TSC (coverage) }\end{array}$ & $\begin{array}{c}\alpha-\text { Mcm3 } \\
\text { TSC (coverage) }\end{array}$ & \begin{tabular}{|c|}
$\lg \mathrm{G}$ \\
TSC (coverage) \\
\end{tabular} & $\begin{array}{c}\alpha-M c m 3 \\
\text { TSC (coverage) }\end{array}$ \\
\hline \multirow{11}{*}{ 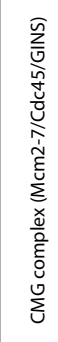 } & $\operatorname{Mcm} 2(100)$ & $364(46 \%)$ & $1348(78 \%)$ & $17(17 \%)$ & 975 (71\%) \\
\hline & Mcm3 (90) & $222(55 \%)$ & $1610(90 \%)$ & $17(20 \%)$ & 1111 (84\%) \\
\hline & Mcm4 (97) & $198(45 \%)$ & 1277 (85\%) & $7(7.4 \%)$ & 947 (75\%) \\
\hline & Mcm5 (82) & $70(59 \%)$ & $720(88 \%)$ & $30(17 \%)$ & 927 (83\%) \\
\hline & Mcm6 (93) & $196(60 \%)$ & 964 (91\%) & $39(14 \%)$ & $888(83 \%)$ \\
\hline & Mcm7 (82) & $160(52 \%)$ & $1082(84 \%)$ & $24(8.9 \%)$ & 905 (73\%) \\
\hline & Cdc45 (66) & $18(26 \%)$ & $187(46 \%)$ & $2(2.6 \%)$ & 277 (54\%) \\
\hline & Psf1 (23) & $3(31 \%)$ & $58(90 \%)$ & 0 & $62(85 \%)$ \\
\hline & Psf2 (21) & 0 & $26(58 \%)$ & 0 & $33(79 \%)$ \\
\hline & Psf3 (24) & $3(15 \%)$ & $55(79 \%)$ & 0 & $55(92 \%)$ \\
\hline & Sld5 (26) & $2(10 \%)$ & $42(81 \%)$ & 0 & $44(64 \%)$ \\
\hline \multirow{24}{*}{ 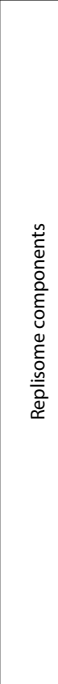 } & Spt16 (118) & $109(23 \%)$ & $431(61 \%)$ & $3(2.9 \%)$ & $453(52 \%)$ \\
\hline & Ssrp1 (79) & $19(8 \%)$ & 224 (45\%) & 0 & $229(48 \%)$ \\
\hline & Ctf4 (97) & $4(4 \%)$ & $82(31 \%)$ & 0 & $166(49 \%)$ \\
\hline & Timeless (149) & $6(3 \%)$ & $112(39 \%)$ & 0 & $171(29 \%)$ \\
\hline & Tipin (40) & 0 & $20(27 \%)$ & 0 & $54(24 \%)$ \\
\hline & Claspin (146) & 0 & 0 & 0 & $72(21 \%)$ \\
\hline & Mcm 10 (95) & 0 & 0 & 0 & $257(44 \%)$ \\
\hline & Ctf18 (113) & 0 & 0 & 0 & $172(36 \%)$ \\
\hline & Ctf8 (14) & 0 & 0 & 0 & $8(52 \%)$ \\
\hline & $\operatorname{Dcc1}(45)$ & 0 & 0 & 0 & $33(38 \%)$ \\
\hline & $\operatorname{Rfc2}(38)$ & 0 & 0 & 0 & $27(54 \%)$ \\
\hline & $\operatorname{Rfc} 3(40)$ & 0 & 0 & $4(14 \%)$ & $36(54 \%)$ \\
\hline & $\operatorname{Rfc} 4(40)$ & 0 & 0 & $2(5.2 \%)$ & $31(57 \%)$ \\
\hline & $\operatorname{Rfc5}(38)$ & 0 & 0 & 0 & $22(56 \%)$ \\
\hline & Pola.1 (165) & 0 & 0 & 0 & $109(30 \%)$ \\
\hline & Prim1 (49) & 0 & 0 & 0 & $14(28 \%)$ \\
\hline & Prim2 (61) & 0 & 0 & 0 & $24(24 \%)$ \\
\hline & Pole1 (261) & $12(5 \%)$ & $166(29 \%)$ & $2(0.88 \%)$ & $543(38 \%)$ \\
\hline & Pole2 (60) & 0 & $15(32 \%)$ & 0 & $103(50 \%)$ \\
\hline & Pole3 (17) & 0 & $4(27 \%)$ & 0 & $10(45 \%)$ \\
\hline & Pol $\varepsilon 4$ (12) & 0 & 0 & 0 & $4(35 \%)$ \\
\hline & Rpa1 (67) & $80(53 \%)$ & $107(48 \%)$ & 0 & $10(12 \%)$ \\
\hline & Rpa2 (29) & $13(22 \%)$ & $30(55 \%)$ & 0 & $2(9 \%)$ \\
\hline & Rpa3 (13) & $6(52 \%)$ & $10(92 \%)$ & 0 & 0 \\
\hline \multirow{4}{*}{ 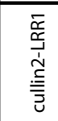 } & cullin2 (87) & $6(8 \%)$ & $4(3 \%)$ & 0 & $124(52 \%)$ \\
\hline & LRR1 (47) & 0 & $11(21 \%)$ & 0 & 46 (44\%) \\
\hline & Elongin $B(13)$ & 0 & 0 & 0 & $5(31 \%)$ \\
\hline & Elongin C (12) & 0 & 0 & 0 & $7(32 \%)$ \\
\hline \multirow{2}{*}{ 可 } & Traip (87) & $2(4 \%)$ & $42(44 \%)$ & 0 & $34(36 \%)$ \\
\hline & RNF213 (47) & 0 & $17(2 \%)$ & 0 & 0 \\
\hline
\end{tabular}

B

Post-termination S-phase replisome

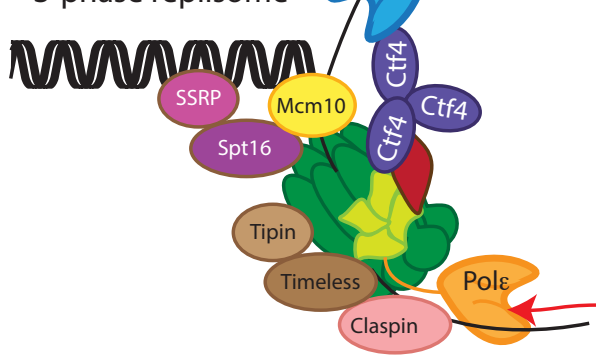

Post-termination mitotic replisome

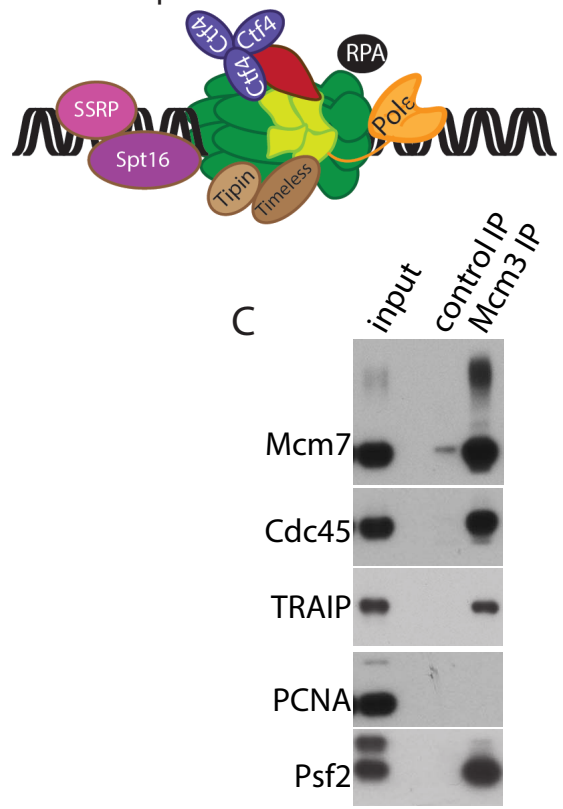




\section{Priego Moreno et al. Fig 3}

A

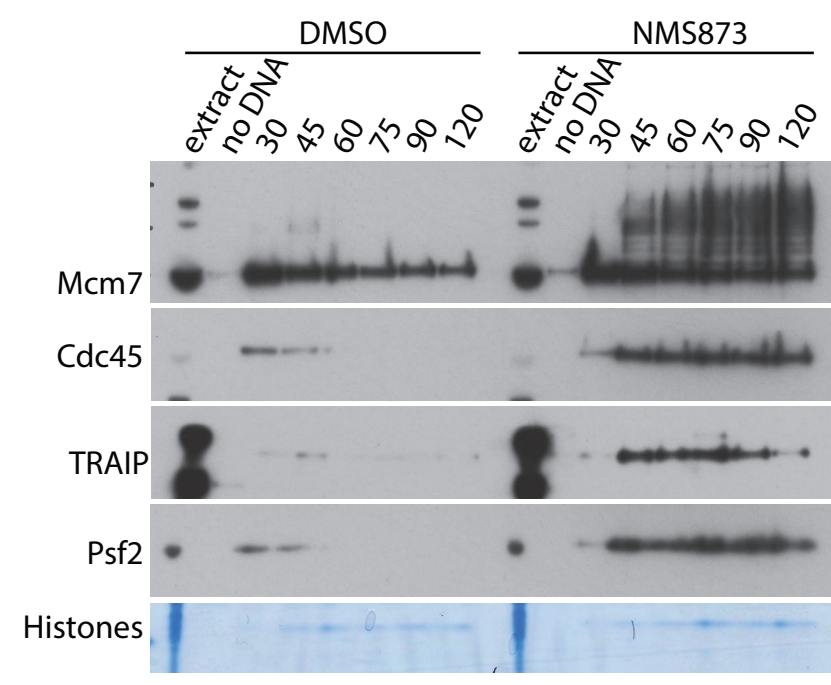

B $[\mathrm{min}]$

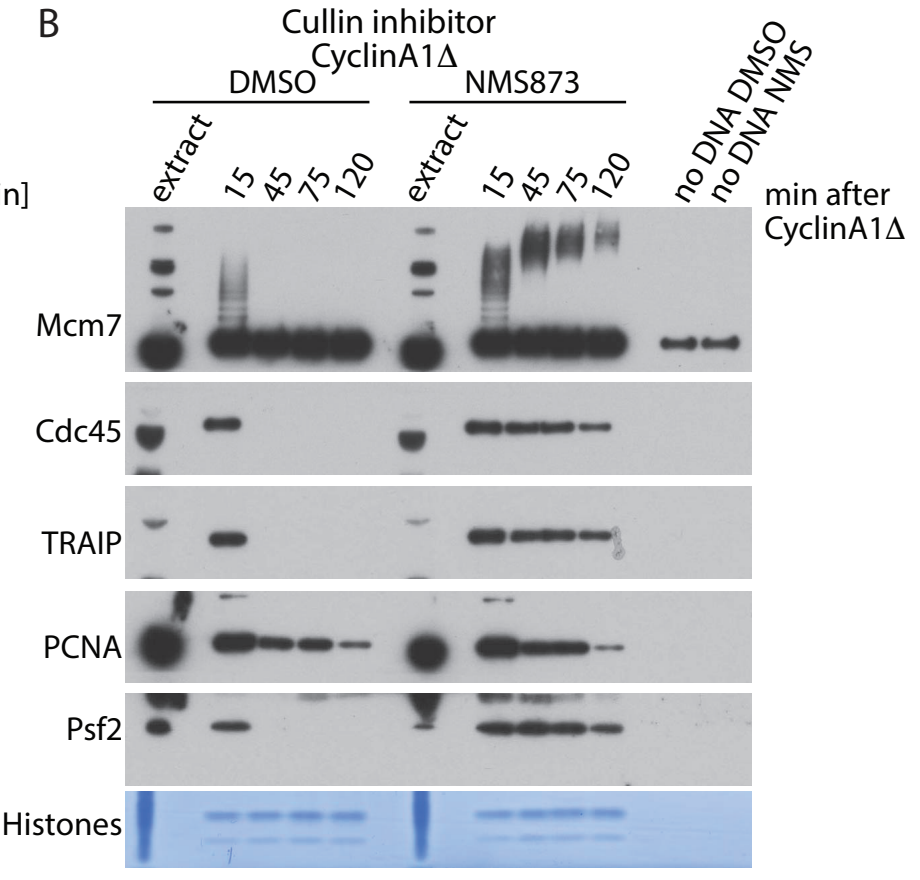

C Cullin inhibitor

CyclinA1 $1 \Delta$

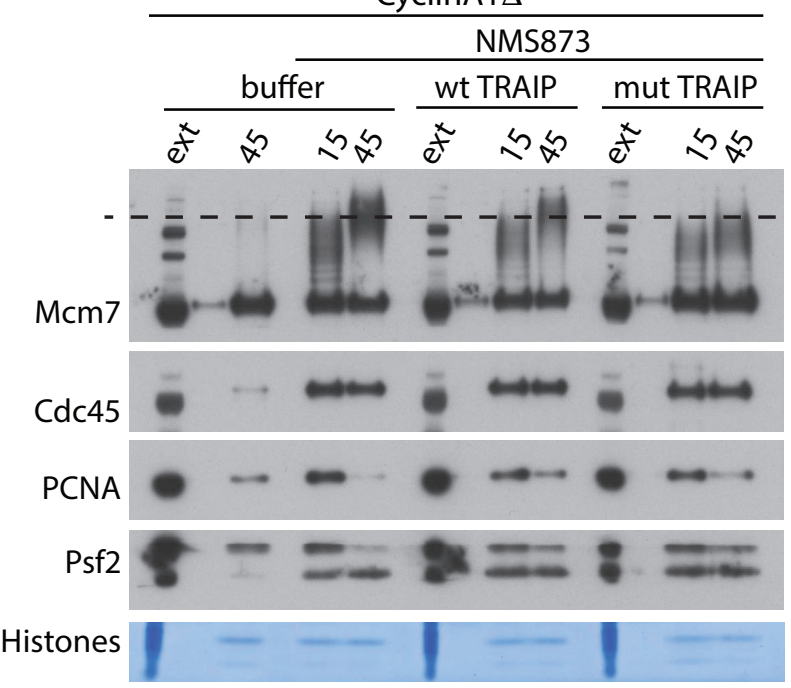

min after CyclinA1 $1 \Delta$ 


\section{Priego Moreno et al. Fig 5}

A

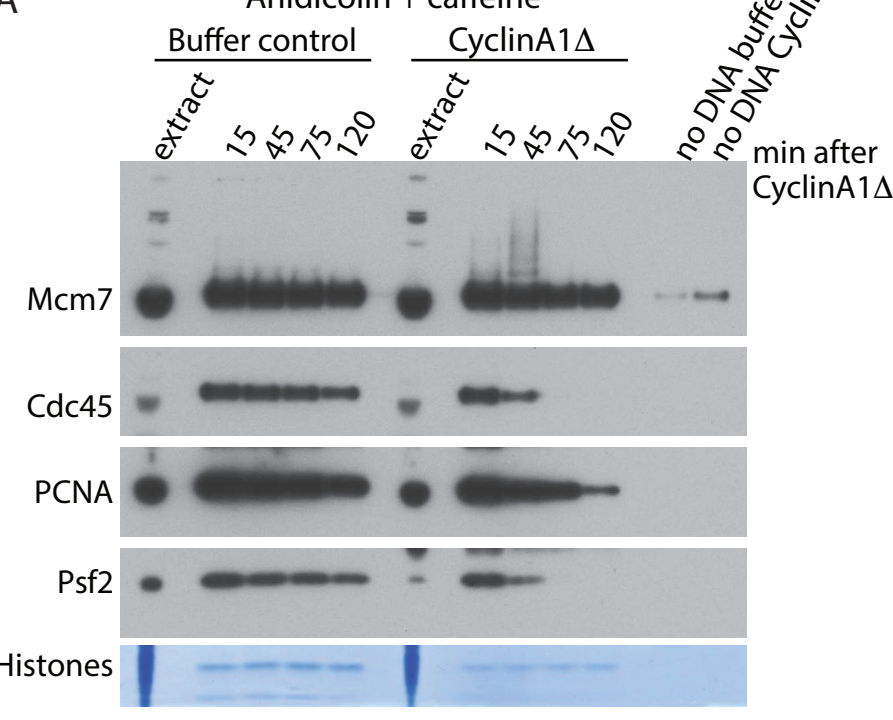

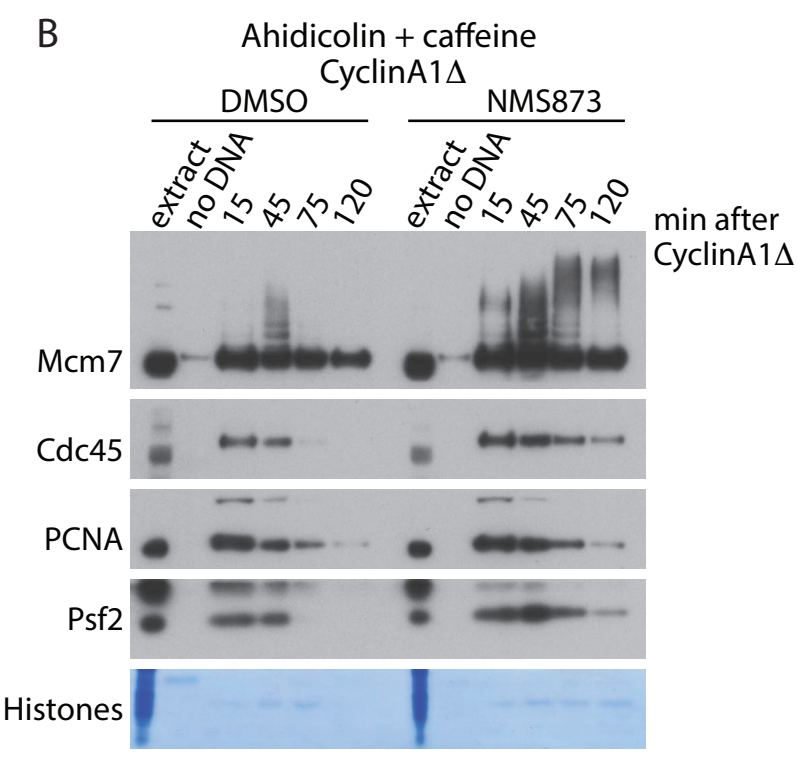




\section{Priego Moreno et al. Fig 4}

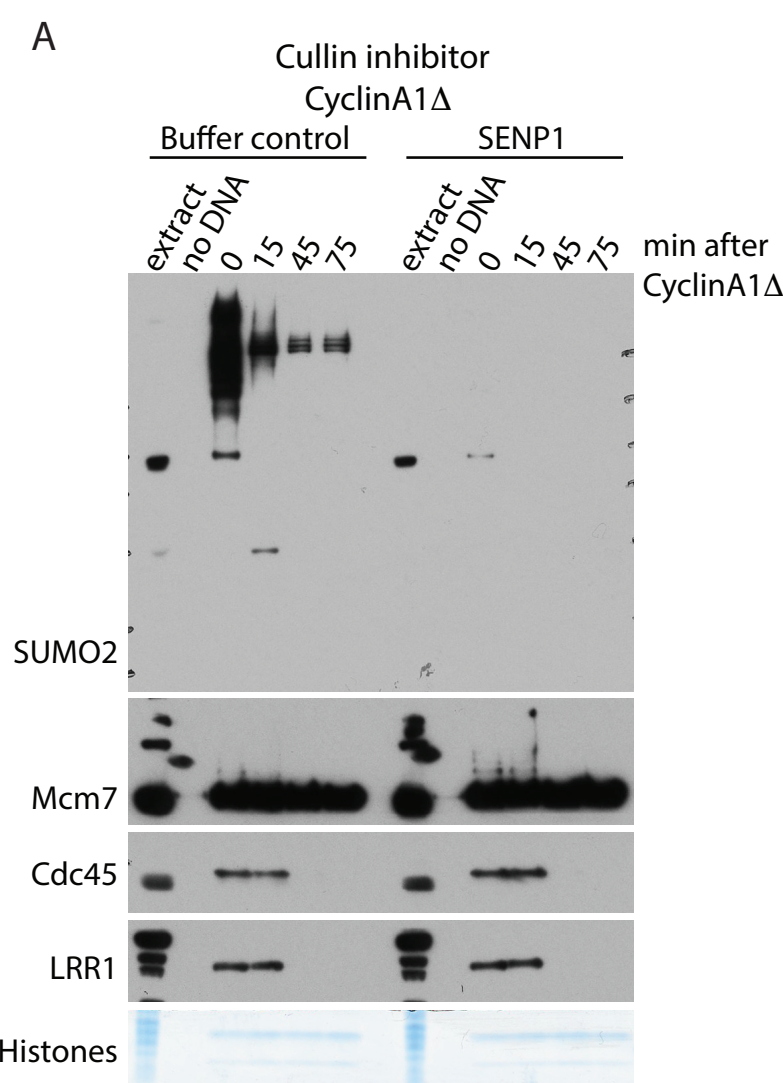

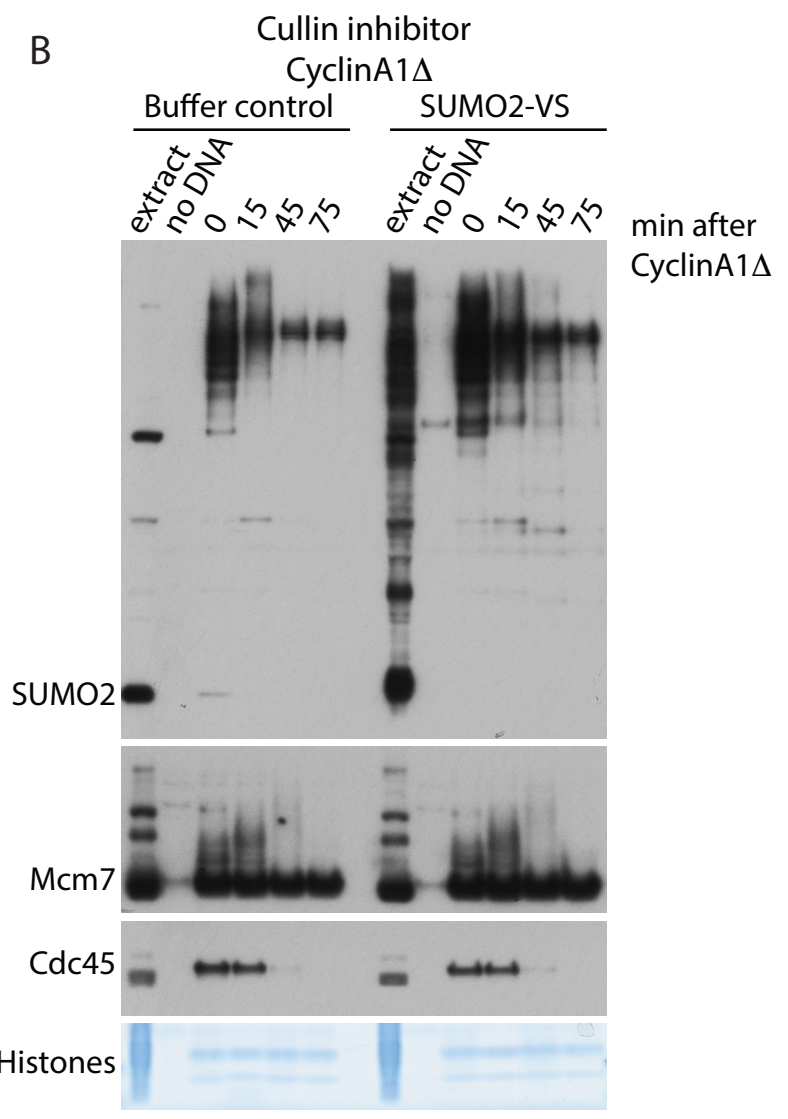


Priego Moreno et al. EV Fig 1

A

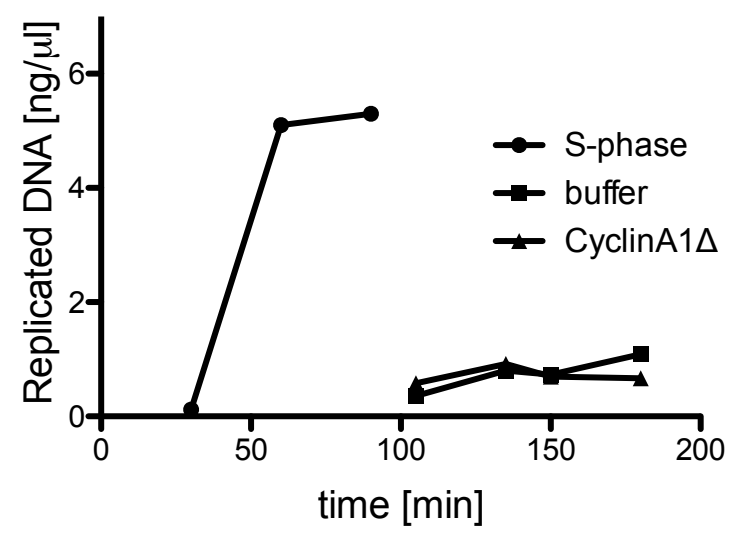

C

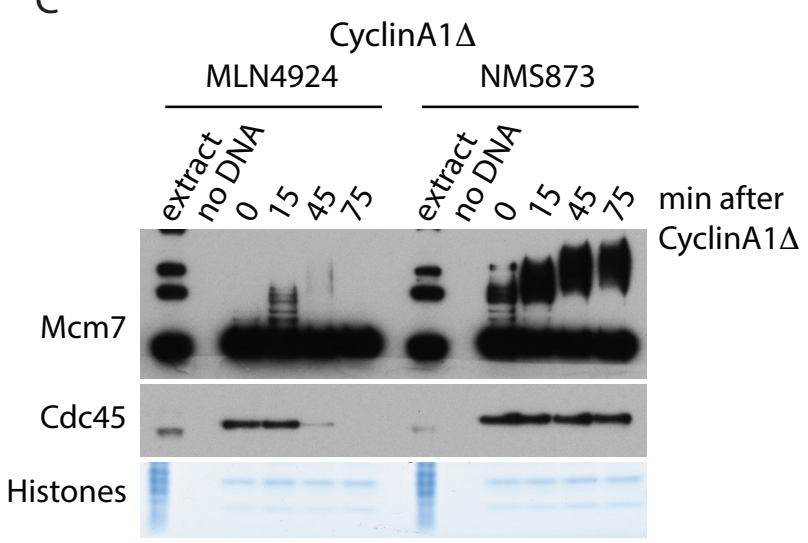

B
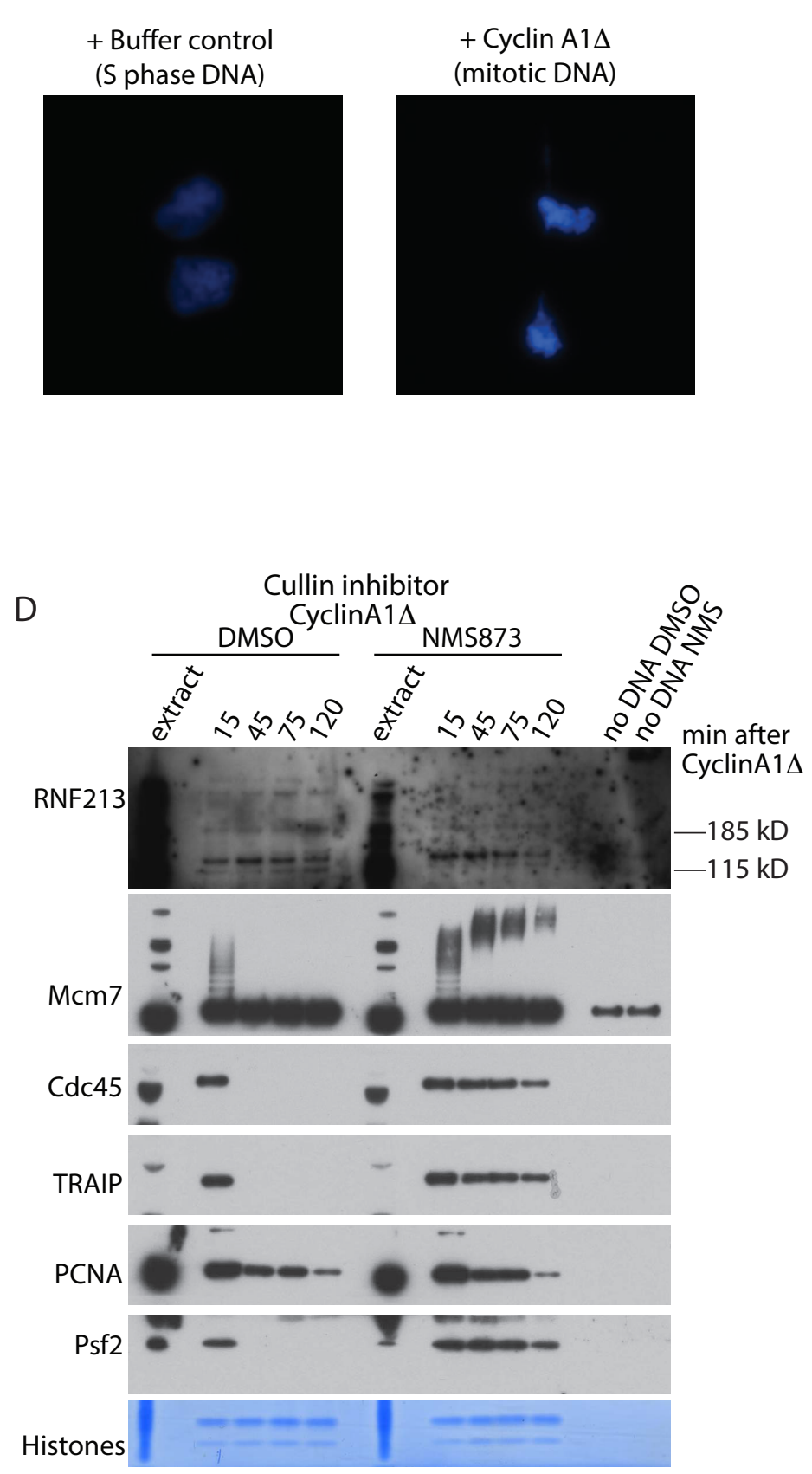
bioRxiv preprint doi: https://doi.org/10.1101/418368; this version posted September 15, 2018. The copyright holder for this preprint (which was not certified by peer review) is the author/funder. All rights reserved. No reuse allowed without permission.

\section{Priego Moreno et al. EV Fig 2}

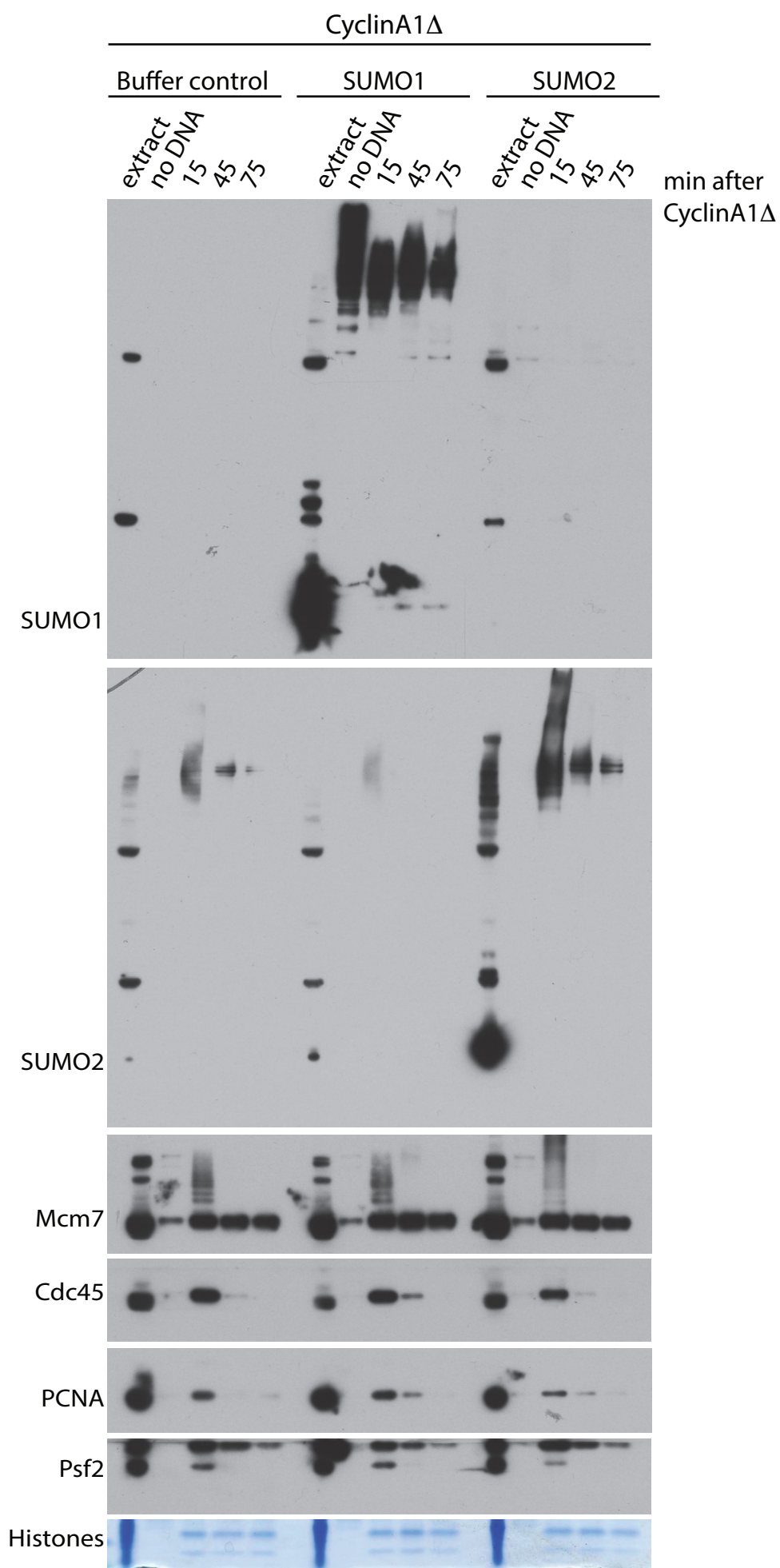


bioRxiv preprint doi: https://doi.org/10.1101/418368; this version posted September 15, 2018. The copyright holder for this preprint (which was not certified by peer review) is the author/funder. All rights reserved. No reuse allowed without permission.

\section{Priego Moreno et al. EV Fig 3}

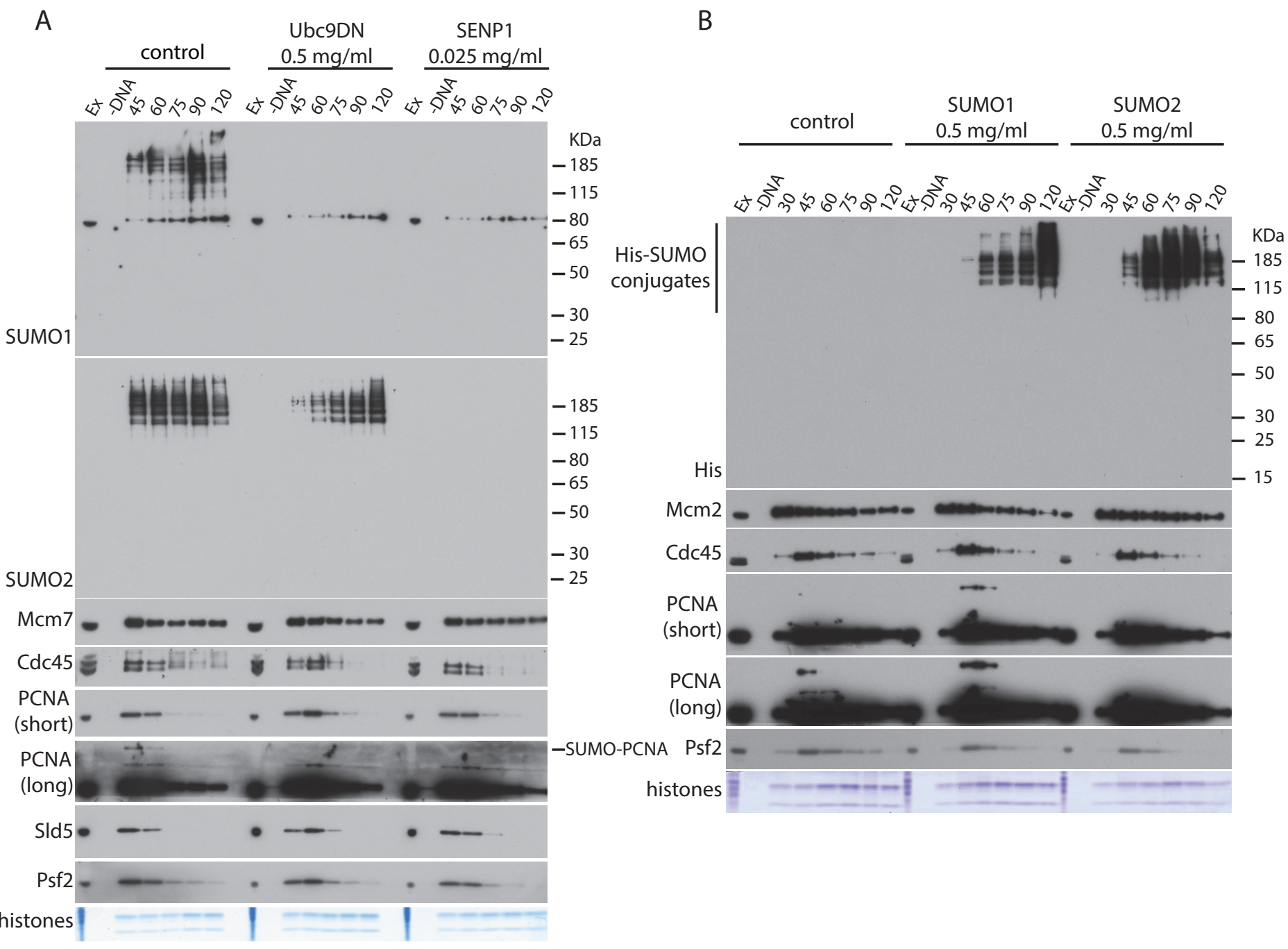

